2 Suppression of HSF1 activity by wildtype p53 creates the driving 3 force for p53 loss-of-heterozygosity, enabling mutant p53 stabilization and invasion

6 Özge Cicek Sener ${ }^{1, \#}$, Adrian Stender ${ }^{1, \#}$, Luisa Klemke ${ }^{1}$, Nadine Stark ${ }^{1}$, Tamara Isermann ${ }^{1}$, 7 Jinyu $\mathrm{Li}^{2}$, Ute M. Moll ${ }^{1,2}$ and Ramona Schulz-Heddergott ${ }^{1, *}$

8

9 1) Institute of Molecular Oncology, University Medical Center Göttingen, 37077 Göttingen, Germany

10 2) Department of Pathology, Stony Brook University, Stony Brook NY 11794, USA

11 * Corresponding author: ramona.schulz@zentr.uni-goettingen.de

$12 \quad$ \# contributed equally to this work

\title{
14 HIGHLIGHTS
}

- heterozygous $\mathrm{p} 53^{\mathrm{R} 248 \mathrm{Q} /+}$ tumors retain $\mathrm{p} 53$ transcriptional activity in a mouse model of colorectal cancer (CRC)

- wildtype p53 actively represses the tumor-promoting HSF1-regulated chaperone system and proteotoxic stress response

- the repressive WTp53 - HSF1 axis creates a selective pressure for WTp53 loss-ofheterozygosity in CRC tumors

- p53 loss-of-heterozygosity enables stabilization of the gain-of-function $\mathrm{p} 53^{\mathrm{R} 248 \mathrm{Q}}$ mutant 


\section{Abstract}

A prerequisite for gain-of-function (GOF) p53 missense mutants (mutp53) is protein stabilization. Moreover, a prerequisite for mutp53 stabilization is loss of the remaining wildtype (WT) p53 allele (loss-of-heterozygosity, p53LOH) in mutp53/+ tumors. Thus, GOF, mutp53 stabilization and p53LOH are strictly linked. However, the driving force for p53LOH is unknown. Typically, heterozygous tumors are an instable transition state. Here we identify the repressive WTp53-HSF1 axis as the driver of p53LOH.

We find that the WTp53 allele in AOM/DSS-induced colorectal tumors (CRC) of p53 ${ }^{\mathrm{R} 248 \mathrm{Q} /+}$ mice retains its haploid transcriptional activity. Notably, WTp53 represses heat-shock factor 1 (HSF1) activity, the master transcription factor of the proteotoxic stress defense response (HSR) that is ubiquitously and constitutively activated in cancer tissues. HSR is critical for stabilizing oncogenic proteins including mutp53. WTp53-retaining murine CRC tumors and tumor-derived organoids and human CRC cells all suppress the tumor-promoting HSF1 transcriptional program.

Mechanistically, the retained WTp53 allele activates CDKN1A/p21, leading to cell cycle inhibition and suppression of the E2F target gene MLK3. MLK3 links cell cycle to the MAPK stress pathway to activate the HSR response. We show that in $\mathrm{p} 53^{\mathrm{R} 248 \mathrm{Q} /+}$ tumors WTp53 activation by constitutive stress (emanating from proliferative/metabolic stresses and genomic instability) represses MLK3, consequently inactivating the MAPK-HSF1 response necessary to ensure tumor survival. This creates strong selection pressure for $\mathrm{p} 53 \mathrm{LOH}$ which eliminates the repressive WTp53-HSF1 axis and unleashes the tumor-promoting HSF1 functions, inducing mutp53 stabilization and enabling invasion.

\section{Keywords} mutp53, HSF1, Hsp90, Hsp70, CDK4, MLK3, MAPK, AOM/DSS, colorectal cancer, organoids, Idasanutlin 
62 Colorectal cancer ( $\mathrm{CRC})$ is due to several driver mutations and the third leading cause of cancer 63 deaths worldwide. TP53 mutations enable the critical transition from late adenoma to invasive carcinoma $^{1,2}$. Next to APC, TP53 mutations are the second most common alteration in sporadic CRC, affecting $>60 \%$ of cases $^{3-10}$. The vast majority of TP53 alterations are missense mutations (mutp53) with hotspot codons R175, G245, R248, R273 and R282 ${ }^{11-13}$. In addition to loss-of-WTp53 function (LOF), some, especially hotspot, mutp53 alleles gain broad tumorigenic gain-of-function (GOF) and actively promote aggressive cancer progression in vivo ${ }^{9,14-20}$. Some GOF mutants acquire allele-specific functions, not necessarily shared by other mutants ${ }^{3,9,21-26}$. The GOF TP53 ${ }^{R 248 Q}$ allele is one of the most common across cancer types ${ }^{10}$.

A prerequisite for GOF is the tumor-specific stabilization of mutp53 proteins by the HSP90/HSP70/HSP40 chaperone systems ${ }^{27-31}$, providing protection from degradation by E3ubiquitin ligases Mdm2 and CHIP ${ }^{32,33}$. HSF1, the master transcription factor of the inducible heat-shock stress response (HSR), governs stress-induced chaperones including HSP90, HSP70 and HSP40 and is the major proteotoxic defense in tumors, preventing aberrant oncoproteins from aggregation ${ }^{34-36}$. Moreover, HSF1 induces chaperone-independent tumorpromoting genes, together imparting on HSF1 a key co-oncogenic role in tumorigenesis ${ }^{37-39}$. Notably, since cancer cells experience cumulative stress during tumorigenesis, HSF1 is increasingly activated ${ }^{40}$.

p53LOH is a critical prerequisite for mutp53 stabilization in tumors. Heterozygous tumors rarely if ever stabilize p53 in vivo, 19, 41, 42. Importantly, the majority of human mutp53 tumors have undergone p53 $\mathrm{LOH}^{43-47}$. Moreover, p53LOH has watershed significance in promoting tumor progression. Recent mouse studies clearly identify p53LOH as strong tumor promoting force ${ }^{41}$, 48, 49. Our previous studies comparing sarcomas and breast cancer identified that heterozygous mutp53 tumors require a second hit for mutp53 stabilization, i.e. loss of the remaining WTp53 allele $^{41}$. However, the driving force behind p53LOH remained elusive.

87 Given how important mutp53 GOF activities are in tumor biology, it is imperative to understand the mechanism that drives stabilization of GOF mutants. The dependency of mutp53 stabilization on p53LOH appears somehow regulated by the remaining WTp53, but its mechanism is unknown. Using a genetically controlled p53LOH system in a CRC model, we show their causal relationship. We identify that the remaining WTp53 allele in $p 53^{R 248 Q /+}$ tumors 
93 and invasion. This creates a strong driving force for $\mathrm{p} 53 \mathrm{LOH}$. In sum, a single pivotal genetic 94 event, p53LOH, simultaneously provides three major evolutionary forces to drive cancer, $i$ ) loss 95 of residual WTp53 suppressor activity including the repressive WTp53-HSF1 axis, ii) tumor96 promoting HSF1 upregulation, and iii) mutp53 protein stabilization which liberates GOF 97 activities. This provides an explanation for the longstanding puzzle why p53LOH strictly 98 correlates with mutp53 stabilization and higher tumor aggressiveness.

99 
103 Stabilization of missense mutant p53 (mutp53) proteins specifically in tumor but not normal cells

104 is a key feature and prerequisite of $\mathrm{GOF}^{16,42}$. Since p53LOH is a critical prerequisite for mutp53

105 stabilization in sarcomas and breast cancer ${ }^{41}$, we examined mutp53 stabilization before and 106 after p53LOH in the colorectal AOM/DSS model ${ }^{9}$. Briefly, we combined the humanized GOF

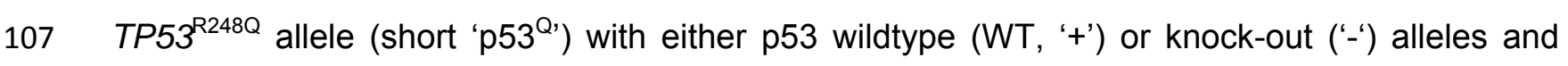
108 determined the p53LOH effect on mutp53 levels (Figures S1A-B). Indeed, massive mutp53 109 stabilization was detected in $100 \%$ of $p 53^{\mathrm{Q} /-}$ tumors, whereas $100 \%$ of $\mathrm{p} 53^{\mathrm{Q} /+}$ tumors failed to 110 undergo stabilization (Figure S1B). Notably, p53LOH increases tumor numbers in the $\mathrm{p}^{-{ }^{--}}$and 111 even more so in the $\mathrm{p} 53^{\mathrm{Q} /-}$ setting (Figure S1C). Notably, 100\% of tumors retaining one WTp53 112 allele $\left(\mathrm{p} 53^{\mathrm{Q} /+}\right.$ and $\left.\mathrm{p} 53^{-/+}\right)$remain noninvasive (Figure S1D). Conversely, loss of the remaining 113 WTp53 allele ( $\mathrm{p} 53^{\mathrm{Q} /-}$ or $\mathrm{p} 53^{-/-}$) enables invasion (Figures S1D-E). Thus, p53LOH is the critical 114 determinant for CRC invasion.

115 To independently validate that p53LOH enables mutp53 stabilization and CRC invasion, we

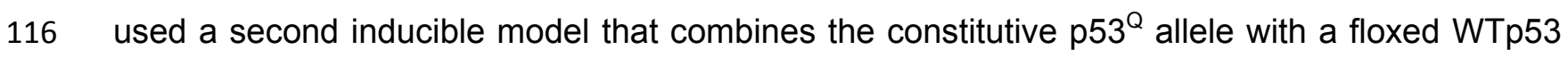
$117\left(\mathrm{p} 53^{\mathrm{fl}}\right)$ allele. $\mathrm{p} 53^{\mathrm{Q} / \mathrm{fl}}$ mice were crossed to villinCreER ${ }^{T 2}$ mice to generate Tamoxifen (TAM)118 inducible $p 53 \mathrm{LOH}$ restricted to intestinal epithelial cells $\left(p 53^{\mathrm{Q} / \Delta}\right)$, plus non-LOH controls $\left(p 53^{\mathrm{Q} /+}\right.$ ) 119 (Figure 1A). Importantly, TAM-mediated p53LOH was induced uniformly at a defined tumor 120 burden verified by colonoscopy (Figure $1 \mathrm{~B})^{50}$. Controls were $(i) \mathrm{p} 53^{\mathrm{Q} / \mathrm{fl}}$ oil-treated mice, and (ii) $121 \mathrm{p} 53^{\mathrm{Q} /+}$ TAM-treated mice to exclude nonspecific TAM effects. At 6-8 wks post TAM, LOH tumors 122 showed a trend towards increased tumor numbers and sizes compared to both 'no LOH' control 123 groups (Figures 1C-D). When analyzed earlier at 3-5 wks post TAM, tumor burden had not yet 124 increased, indicating that LOH's effect on promoting proliferation requires time and is 125 incremental (Figure 1E).

126 Mutp53 stabilization is a critical prerequisite for mutp53 GOF$^{9}$. Indeed, all p53LOH mice 127 exhibited stabilized mutp53, in sharp contrast to mice with a retained WTp53 allele (Figure 1F). 128 The WTp53 allele is a major barrier to tumor invasion as reported by us and others ${ }^{7,9}$, 42. In 129 agreement, 'no LOH' mice (oil-treated $\mathrm{p} 53^{\mathrm{Q} / \mathrm{fl}}$ and TAM-treated $\mathrm{p} 53^{\mathrm{Q} /+}$ mice) never developed 130 invasive tumors (Figure 1G). In stark contrast, induced p53LOH caused a dramatic increase in 131 invasive tumors in the cohort from 0/27 tumors to 18/49 tumors (Figures $1 \mathrm{G}-\mathrm{I}$ ) and all mice 132 harbored at least one invasive tumor. Notably, mutp53 stabilization is particularly prominent at 133 the invasive front (Figure 1I). Moreover, in the constitutive p53LOH model, tumors lacking 
WTp53 confirmed the dramatic increase in invasion (Figures S1D-E). In sum, while LOH only has an incremental effect on tumor proliferation, p53LOH is a dramatic gate-opener unleashing GOF by mediating mutp53 stabilization, which in turn enables invasion.

\section{The WTp53 allele in heterozygous colorectal tumors retains its activity and suppresses the HSF1 transcriptional program}

While mutp53 stabilization after p53LOH is dramatic, the mechanism of tumor-specific mutp53 accumulation triggered by $\mathrm{p} 53 \mathrm{LOH}$ is incompletely understood. In agreement with other studies $^{16,51}$, loss of Mdm2 induction by the WT allele might play some role (Figure 2A, compare Mdm2 mRNA in $p 53^{\mathrm{Q} /+}$ vs $p 53^{\mathrm{Q} /-}$ tumors). However, an additional mechanism likely exists to ensure such massive stabilization after p53LOH.

A major pathway for tumor-specific mutp53 stabilization is the intrinsic tumor stress-induced HSF1-governed chaperone system ${ }^{14}, 33,35,52,53$. In cancer cells the constitutively (phospho-) activated master transcription factor HSF1 orchestrates the major proteotoxic defense. Thus, we asked whether in heterozygous tumors the remaining WTp53 suppresses global HSF1 activity or distinct chaperone targets. This hypothesis assumes that despite the presence of a GOF allele ( $Q$ in this case), the remaining WTp53 allele at least partially retains its transcriptional activity. Thus, we treated tumor-bearing $p 53^{\mathrm{Q} /+}$ mice with Nutlin, a highly specific non-genotoxic p53 activator inhibiting its E3 ligase MDM2, to mimic the general activation state of WTp53 in tumors constitutively stressed by aberrant growth and metabolic stress, hypoxia and genomic instability (Figure 2B).

Indeed, in $\mathrm{p} 53^{\mathrm{Q} /+}$ tumors Nutlin induced allele-dose dependently (haploid) WTp53 target gene expression (e.g. Cdnk1a, Gadd45a and Sfn) (Figure 2C). Interestingly, however, Mdm2

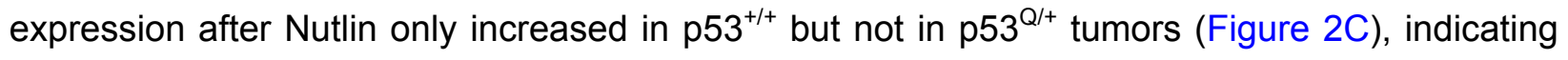
that p53-regulated Mdm2 levels cannot account for the missing mutp53 stabilization in heterozygous tumors. Why Mdm2 failed to increase remains unclear. We conclude that, surprisingly, the GOF mutp $53^{\mathrm{R} 248 \mathrm{Q}}$ allele fails to exert a dominant-negative effect over the remaining WTp53 allele as predicted by many, mainly in vitro, studies ${ }^{54-57}$. Importantly, this residual WTp53 activity is sufficient to suppress canonical HSF1 target genes in $\mathrm{p} 53^{\mathrm{Q} / \mathrm{t}}$ tumors

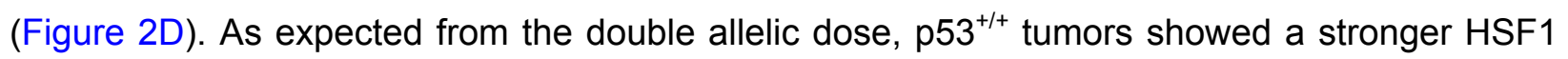
target gene suppression after Nutlin (Figure 2D). 
165

166

167

168

169

170

171

172

173

174

175

176

177

178

179

180

181

182

183

184

185

186

187

188

189

190

191

192

193

194

195

We next tested whether simple loss of the WTp53 allele is able to activate HSF1 without Nutlin. While p53-/- vs. p53+/+ AOM/DSS mice have accelerated tumor growth (larger tumor numbers and sizes, Figures S2A-D) due to reduced cell cycle inhibitory/pro-apoptotic p53 target gene expression (Figures S2E-F), only some HSF1 target genes increased (Figure S2G). Conversely, stress-activated WTp53, mimicked by Nutlin, suppresses HSF1 activity to prevent chaperonemediated mutp53 stabilization (Figure 2D).

In sum, in a stressed tumor milieu activated WTp53 in heterozygous mutp53/+ tumors creates the driving force for p53LOH. p53LOH eliminates the repressive WTp53-HSF1 axis and enables activation of the broad co-oncogenic HSF1 functions, which causes mutp53 protein stabilization that in turn enables tumor growth but foremost invasion.

\section{Activated WTp53 represses HSF1 activity in human colorectal cancer cells}

Since mutp53 stabilization specifically arises in the malignant epithelial compartment, we analyzed the mechanism of p53-mediated HSF1 suppression in human CRC cell lines harboring WTp53. We resorted to homozygous WTp53 lines because heterozygous human CRC lines are not readily available. Importantly, measuring the global HSF1-mediated HSR response by heatshock response element (HSE) luciferase assay confirmed HSF1 suppression upon WTp53 activation by Nutlin (Figure 3A). Moreover, Nutlin-induced HSF1 suppression was rescued by shp53-mediated depletion, confirming that the Nutlin-induced effect is p53-specific (Figure 3B).

HSF1 not only orchestrates the cellular chaperone system. In cancer cells HSF1 also broadly upregulates a large palette of tumor-promoting genes involved in cell cycle, DNA repair, metabolism, adhesion and protein translation ${ }^{34}$. Thus, we analysed randomly selected HSF1 targets representing different functions (Figure $3 \mathrm{C}$ ). Notably, upon p53 activation by Nutlin we observed repression of classic HSF1 targets including HSP90AA1, HSPA1A, HSPH1 and HSPB1, validating the mouse model (Figure $3 C$ ). Moreover, Nutlin also suppressed the tumorpromoting HSF1 targets CDC6, ITGB3BP, RBBP5, BST2 and FBLN1 (Figure 3C). Importantly, p53 depletion by siRNAs rescued their repression, confirming that p53 specifically regulates HSF1 activity (Figures 3D, S3A). The critical phosphorylation site for HSF1 activation is residue Ser326 which serves as functional hallmark of the tumor-promoting HSR response ${ }^{37,58 .}$ Concomitantly to HSF1 target gene repression, p53 activation profoundly reduced pSer326HSF1 levels in HCT116, RKO (Figure 3E), LS513 and LS174T cells (Figure S3B). Again, p53 
196

197

198

199

200

201

202

203

204

205

206

207

208

209

210

211

212

213

214

215

216

217

218

219

220

221

222

223

224

225

226

depletion by siRNAs (Figure S3C) or p53 deletion in isogenic HCT116 cells (Figure S3D) abolished pSer326-HSF1 dephosphorylation. Conversely, mutp53-haboring CRC cells failed to repress HSF1 after Nutlin (Figure S3E). Of note, total HSF1 protein remained unchanged, excluding that HSF1 dephosphorylation/ inactivation is simply a consequence of reduced total HSF1 levels (Figure S3C). Consequently, HSF1 inactivation reduced heat-shock protein expression such as Hsp90a and Hsp27 (Figure 3F). Moreover, HSP90 clients including c-Raf, AKT and Bcl-xl also destabilized, confirming the inactivation of the HSF1-HSP90 antiproteotoxic defense response upon p53 activation (Figure 3F).

To further strengthen the evidence for this repressive WTp53-HSF1 axis, we generated stable HSF1-overexpressing HCT116 clones, functionally confirmed by increased levels of pSer326HSF1 (Figure 3G) and higher expression of HSF1 target genes (Figure S3F). Again, Nutlin strongly dephosphorylated pSer326-HSF1 (Figure 3G) and down-regulated the increased HSF1 target gene response in these clones (Figure S3F). To finally demonstrate that HSF1 is directly controlled by WTp53, we used heat-shock, the strongest known HSF1 activator, to massively increase endogenous HSF1 activity in human CRC cells. Again, Nutlin strongly repressed HSF1 activity, demonstrating how potently WTp53 counter-regulates even the strongest HSF1 activator (Figures $3 \mathrm{H}-\mathrm{I}$ ). In sum, the HSF1-mediated stress response is strongly attenuated by activation of WTp53.

\section{p53 blocks HSF1 activity via p21-mediated cell cycle inhibition in human CRC cells}

To gain further insight into Nutlin-induced HSF1 repression, we analyzed CDKN1A/p21, a key p53 target gene and potent cyclin-dependent kinase inhibitor that mediates cell cycle arrest (Figure S4A). Indeed, p21 depletion by siRNAs abolished pSer326-HSF1 dephosphorylation (Figure 4A) and nearly reversed Nutlin-induced HSF1 target gene repression (Figures 4B, S4B), indicating a p53-p21-mediated HSF1 suppression.

Next we asked whether the p53/p21-mediated HSF1 suppression is linked to and regulated by the cell cycle. CDKN1A/p21 binds and inhibits cyclin-dependent kinases (CDKs), thereby preventing phosphorylation of the retinoblastoma protein (RB). Hypo-phosphorylated RB binds to and inhibits E2F transcription factors preventing S-phase entry ${ }^{59}$. Thus, we tested whether cell cycle inhibitors like CDK4/6 inhibitor Palbociclib phenocopy the p53-p21-mediated HSF1 inactivation. Indeed, both Nutlin- and Palbociclib-treated cells exhibited markedly decreased 
227 levels of pSer326-HSF1 in WTp53 cells (Figure 4C). Moreover, HSF1 targets were suppressed 228 by Palbociclib, mimicking the Nutlin-induced HSF1 response (Figure 4D). In further support, 229 Nutlin-derivatives RG7112 and RG7388 (Idasanutlin) also reduced pSer326-HSF1 (Figures 4E230 F). Likewise, in HSF1-overexpressing HCT116 clones cell cycle inhibition by Palbociclib (like 231 Nutlin) repressed pSer326-HSF1 levels (Figure 4G) and target gene expression (Figure 4H).

232 To pinpoint the specific CDKs involved in activating HSF1, we used RO3306 (inhibits CDK1 and 233 CDK2 at lower concentrations but CDK4 at higher concentrations) and Roscovitine (inhibits 234 CDK1, CDK2, CDK5 and CDK7, but poorly CDK4/CDK6). Of note, only RO3306 at higher 235 concentrations blocked pSer326-HSF1 like Nutlin and Palbociclib did (Figure 4I), indicating a 236 specific role for CDK4/6 in HSF1 activation. Overall, these data demonstrate that cell cycle 237 inhibition via p53-induced p21-CDK4/6 signaling suppresses HSF1 activity.

WTp53 activation represses MLK3. MLK3 links cell cycle to the MAPK stress pathway to activate the HSF1 response

241 The HSF1 stress response is markedly attenuated by CDK4/6 inhibition (Figure 4). Thus, we 242 tested whether E2F target genes like CDK1, CDK2, CDC25C, PLK4 and MLK3 control the 243 HSF1-mediated HSR. Indeed, these E2F targets are all strongly repressed by Nutlin-activated 244 p53, an effect largely rescued by concomitant p53 depletion (Figure 5A). Specifically, MLK3 245 depletion mimicked the Nutlin response and reduced both pSer326-HSF1 (Figure 5B) and HSF1 246 target gene expression (Figures 5C-D). Notably, MLK3 directly signals to the MEK/ERK stress 247 pathway $^{60-62}$ and MEK/ERK activates HSF1 by phosphorylation ${ }^{35,63,64}$. In contrast, depletion of 248 CDK1 or CDK2 failed to reduce pSer-325 HSF1 (Figures S5A-B). Moreover, PLK4 protein was 249 not diminished after silencing, albeit PLK4 mRNA was strongly reduced, pointing to a stable 250 PLK4 protein but excluding PLK4 as HSF1-activating kinase (Figures S5C, D). In contrast, 251 MLK3 depletion reduced both pSer326-HSF1 and MEK phosphorylation (Figure 5B), revealing a 252 MLK3-MEK-HSF1 signaling axis.

253 Importantly, MLK3 mRNA and protein levels were reduced after p53 activation (Nutlin and 254 RG7112, Figures 5A-B, E-F, S5E) and cell cycle inhibition (CDK4i and RO3306, Figures 5E-G), 255 concomitant with MEK inactivation (pMEK1, Figures 5E-G). Thus, we confirmed the MAPK 256 pathway as major HSF1 activator in human WTp53 harboring CRC cells. Taken together, we 257 identified MLK3 as upstream link between cell cycle and the MAPK stress pathway to activate 
258

259

260

261

262

263

264

265

266

267

268

269

270

271

272

273

274

275

276

277

278

279

280

281

282

283

284

285

286

287

288

289

HSF1. WTp53 activation represses MLK3, which in turn inactivates the MAPK stress pathway and consequently the HSF1 response.

\section{In human colorectal cancer p53LOH combined with missense mutp53 tends to shorten} patient survival and upregulate HSF1 activity

Tumors strongly depend on constitutively upregulated chaperones to manage pervasive proteotoxic stress. However, functional WTp53 prevents adaptive upregulation of the HSF1 chaperone system (Figures 2-5). Thus, to survive and progress, tumors are under strong selection pressure to undergo p53LOH ${ }^{65}$ and lose WTp53-mediated HSF1 repression. This scenario was confirmed in human CRC. p53LOH occurred in $\sim 80 \%$ of patients harboring all p53 variants or missense-only (MS) (Figure 6A, COADREAD TCGA data). Importantly, p53LOH combined with missense mutp53 showed a trend to shorter survival (median 57.2 month vs. 83.2 months in WTp53 patients) (Figure 6B, note that TCGA lacks sufficient numbers of heterozygous patients (mutp53/+), precluding statistical analysis). Remarkably, HSF1 target genes are concomitantly upregulated in p53LOH CRCs harboring all p53 mutations (Figure S6A) or missense-only variants (Figure 6C). Moreover, p53LOH breast cancers (BRCA TCGA) also exhibited upregulated HSF1 targets (Figures S6B-C). Furthermore, BRCA cells repressed pSer326-HSF1 when harboring WTp53 but not mutp53 (Figure S6D). In sum, these data support that in human colorectal and breast cancers p53LOH overrides HSF1 repression by WTp53 and enables pleiotropic tumor-promoting HSF1 functions contributing to poorer prognosis.

\section{In murine CRC organoids p53LOH enables HSF1 activity and triggers mutp53 stabilization}

To further strengthen the WTp53-induced HSF1 repression in heterozygous epithelial cells, we generated tumor-derived organoids from our murine CRCs (Figure 7A). Importantly, p53 ${ }^{\mathrm{Q} / \mathrm{fl}}$ organoid cultures maintain their heterozygous p53 status over at least 8 passages (Figures S7A-B). Thus, we treated $\mathrm{p} 53^{\mathrm{Q} / \mathrm{fl}}$; vilCreER ${ }^{\mathrm{T} 2}$ tumor-derived organoids first with $4 \mathrm{OHT}$ to induce p53LOH, followed by Nutlin (Figures 7A-B). Heterozygous organoids (EtOH controls) showed strong induction of p53 target genes after Nutlin, whereas the p53 response was significantly 
290 damped in p53LOH organoids (4OHT group) (Figure 7B). Cre recombinase-mediated allele

291 deletion is never $100 \%$ efficient, creating competition between non-recombined and recombined

292 tumor cells. Indeed, p53 mRNA levels post 4OHT were above those corresponding to a single

293 (mutant) TP53 copy (Figure S7C), indicating that recombination was below 100\%, and

294 explaining the mild but detectable Nutlin response in 4OHT-treated organoids (Figure 7B).

295 Importantly, HSF1 target genes were de-repressed in p53LOH organoids (4OHT group) after

296 Nutlin versus the no-LOH (EtOH) group (Figure 7C), again confirming that p53LOH enables

297 HSF1 activity.

298

299 In agreement with an upregulated chaperone system after p53LOH, nuclear mutp53 ${ }^{\mathrm{R} 248 \mathrm{Q}}$ 300 became strongly stabilized after 4OHT (Figures 7D-E). Moreover, while WTp53 strongly 301 prevents invasion by upregulating e.g. miR34a which controls epithelial-to-mesenchymal 302 transition $(E M T)^{66}$, miR34a induction is markedly diminished after p53LOH, allowing 303 upregulation of EMT markers Vimentin and Snail (Figure 7F, compare EtOH+Nutlin vs $3044 \mathrm{OHT}+$ Nutlin). Combined upregulation of EMT genes and pro-invasive HSF1 target genes like $305 \mathrm{HspH}^{67}$ and $\operatorname{ltgb3bp}^{39}$ (Figure $7 \mathrm{C}$ ) enable invasiveness in a stressed tumor milieu following $306 \mathrm{p} 53 \mathrm{LOH}$.

307 


\section{DISCUSSION}

310

311

312

313

314

315

316

317

318

319

320

321

322

323

324

325

326

327

328

329

330

331

332

333

334

335

336

337

338

339

340

341

Here we use an autochthonous immune-competent mouse model that recapitulates human colorectal cancer $^{68}$ and identify the mechanism that drives the critical p53LOH event in p53 missense mutant heterozygous tumors. We show that the GOF $p 53^{\mathrm{R} 248 \mathrm{Q}}$ allele fails to exert a dominant-negative effect over the remaining WTp53 allele. Instead, the activated WT allele retains its tumor-suppressive function and strongly suppresses the potent HSF1-mediated stress response necessary for tumor maintenance and progression. We identify a repressive WTp53-p21-MLK3-MAPK-HSF1 signaling cascade as the underlying mechanism that creates the driving force for losing the WTp53 allele. The retained WTp53 allele, via its ability to repress the HSF1-regulated chaperone system, prevents stabilization of mutp53 protein in heterozygous tumors, thereby blocking the full oncogenic potency of GOF alleles. Hence, WTp53-mediated HSF1 suppression exerts a strong selection pressure for p53LOH. Conversely, p53LOH, once it occurs, is a dramatic all-or-none gate-opener, unleashing the broad GOF functions of the mutant allele by mediating mutp53 stabilization via upregulating the HSF1-HSP90 chaperone system. This in turn enables tumor invasion ${ }^{8,9,16,69}$. Our findings, corroborated by CRC murine organoids, human cell lines and human tumors in which mutp53 enables the critical transition from late adenoma to invasive carcinoma ${ }^{1,2}$, reveal the pivotal significance of the repressive WTp53-HSF1 axis. Thus, a single genetic event, p53LOH, kills 3 birds with one stone: $i$ ) losing WTp53 suppressor activity including HSF1 repression, ii) upregulating tumor-promoting HSF1, and iii) enabling mutp53 protein stabilization, thereby unleashing the GOF potential.

Studies of normal tissues of mutp53 knockin mice established that MDM2 degrades mutant and WTp53 equally well, keeping both mutant and WT levels below immunohistochemical detection $^{16,18,42}$. Conversely, in response to stress both WT and mutp53 proteins stabilize ${ }^{56,70,}$ 71. Importantly, we find in our CRC model that the remaining WT allele in heterozygous tumors is fully activatable, excluding a dominant-negative effect (DNE) by the counterpart mutp53 allele. Although DNE is a likely key driver behind the p53 mutational spectrum in myeloid malignancies ${ }^{72}$, many missense mutants are highly inefficient in their ability to exert DNE in epithelial carcinomas and sarcomas, including the hotspot $p 53^{\mathrm{R} 248 \mathrm{Q}}$ allele ${ }^{9,31,56,73}$. We and others speculate that for DNE to occur in solid tumors, the MUT/WT protein ratio has to greatly shift in favor of $\mathrm{MUT}^{56,74}$. Yet, while DNE requires acute stress to increase the low-abundance mutp53 protein and stoichiometrically overwhelm co-expressed WTp53, stress equally stabilizes WTp53 levels, thus not shifting the ratio. Only a highly active HSF1-chaperone system with its 
342 mutp53-selective accumulation can induce the required MUT predominance over WT. However,

343 the repressive WTp53-HSF1 axis prevents such unilateral mutp53 stabilization, explaining the

344 strong selection pressure for $\mathrm{p} 53 \mathrm{LOH}$.

345

346

AOM/DSS CRCs require WTp53 activation by Nutlin (mimicking high proliferative stress or 347 chemotherapy) to fully regulate HSF1 (Figures 2D, S2G), patient p53LOH tumors intrinsically 348 exhibit upregulated HSF1 targets compared to WTp53 tumors (Figures 6C, S6A-C). We 349 speculate that heterozygous human tumors are sufficiently constitutively stressed to activate 350 WTp53 and repress HSF1, and that upon p53LOH human tumors massively upregulate HSF1 351 activity. In contrast, we posit that baseline AOM/DSS-induced tumors have insufficient stress 352 levels to drive p53LOH spontaneously, explaining the missing spontaneous p53LOH in the 353 AOM/DSS model (Figures 1F, S1B), compared to KRAS-driven mouse models of pancreas and 354 lung cancer ${ }^{42,45}$. Notably, in human CRCs strong constitutive oncogenic stress from K-RAS/ 355 EGFR/ TGF $\beta R /$ PDGFR mutations are preeminent ${ }^{65,69,75,76}$, while AOM/DSS tumors undergo predominantly CTNNB1 but no K-Ras and other proliferative driver mutations ${ }^{68,77}$ which promote proliferative stress ${ }^{65}$. Thus, baseline murine CRCs might not be stressed enough to spontaneously activate WTp53 and suppress HSF1 (Figure S2G). This might also explain the preferred order in which cancer-causing mutations occur during tumorigenesis ${ }^{76,78}$.

Heat shock-induced accumulation and activation of WTp53 also depends on HSF1 $1^{40,79,80}$ and HSF1/chaperone functions ${ }^{71,81-84}$. Here we identify a hitherto unknown repressive feedback WTp53-HSF1 counter-regulation. Our data provide an explanation for the longstanding puzzle why tumor heterozygosity tends to be unstable and why p53LOH strictly correlates with mutp53 stabilization and higher tumor aggressiveness. 
374 We thank Nina Pfisterer and Lukas Gebauer (Molecular Medicine MSc-Program Göttingen) for 375 technical assistance. R.S.-H. is supported by the DFG (SCHUH3160/3-1), the Heidenreich-von376 Siebold Program (University Medical Center Göttingen) and the KH-Bauer Program (G-CCC 377 Göttingen). U.M.M. is supported by $\mathrm{NIH} \mathrm{NCI} \mathrm{(2R01CA176647),} \mathrm{Deutsche}$ 378 Forschungsgemeinschaft (MO1998/2-1) and the Stony Brook Foundation TRO program.

379 
Figure 1. p53 loss-of-heterozygosity (p53LOH) is a prerequisite for mutp53 protein stabilization and enables invasion in colorectal cancer

385

386

387

388

389

390

391

392

393

394

395

396

397

398

399

400

401

402

403

404

405

406

407

408

409

410

411

412

413

414

415

416

417

(A)p53LOH induction scheme in intestinal epithelial cells. The constitutive GOF TP53 ${ }^{\mathrm{R} 248 \mathrm{Q}}$ allele $\left(\mathrm{p} 53^{\mathrm{Q}}\right)$ is paired with the conditional wildtype Trp53 allele $\left(\mathrm{p} 53^{\mathrm{fl}}\right)$ harboring loxP sites in Introns 2 and 10 for Tamoxifen (TAM)-induced Cre recombinase-mediated deletion. A constitutive WTp53 allele ('p53 ${ }^{+\prime}$ ) serves as control. Colorectal tumors were initiated by a bolus of AOM/DSS at age 10 wks. Tumor burden was visualized by weekly colonoscopy (colos). At a defined tumor burden (when heterozygous mice had at least 2-3 S2 tumors and at least one S3 tumor), TAM

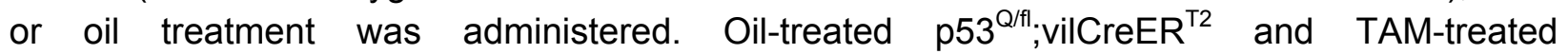
${\mathrm{p} 53^{\mathrm{Q} /+} ; \text { vilCreER }}^{\mathrm{T} 2}$ control mice carry heterozygous tumors, whereas TAM-treated $\mathrm{TP}^{\mathrm{R} 248 \mathrm{R} / \mathrm{fl}} ;$ vilCreER $^{\mathrm{T} 2}\left(\mathrm{p} 53^{\mathrm{Q} / \Delta}\right)$ mice carry p53LOH tumors. TAM-treated TP53 ${ }^{\mathrm{R} 248 \mathrm{Q} /+} ;$ vilCreER $^{\mathrm{T} 2}$ mice served as additional control to exclude nonspecific TAM effects. Mice were analyzed 2-8 wks after LOH induction.

(B) Representative colonoscopy image of an untreated TP53 ${ }^{\mathrm{R} 248 \mathrm{Q} / \mathrm{fl}} ; \mathrm{vilCreER}^{\mathrm{T} 2}$ mouse at 8 wks post AOM. Tumors with scores S2 (solid arrow) and S3 (dashed arrows) ${ }^{50}$.

(C) Representative macroscopic view of the entire dissected colons of $\mathrm{p} 53^{\mathrm{Q} / \mathrm{fl}}$ mice treated as indicated, 6 wks after inducing p53LOH. Left end, ileocecal valve; right end anus.

(D) Number of colonic tumors per mouse (left) and tumor size distribution (right) of the indicated genotypes analyzed at 6-8 wks post TAM or oil treatment. Note that both 'no LOH' groups had the same tumor burden. $p 53^{\mathrm{Q} / \mathrm{fl}}+$ oil group with $\mathrm{n}=6, \mathrm{p} 53^{\mathrm{Q} /+}+$ TAM group with $\mathrm{n}=5, \mathrm{p} 53^{\mathrm{Q} / \mathrm{fl}}+$ TAM group with $n=8$. Mean \pm SEM, Student's t-test. $p^{*}=0.05$; $n s$, not significant.

(E) Number of colonic tumors per mouse (left) and tumor size distribution (right) of the indicated genotypes analyzed earlier than in (D) at 3-5 wks post TAM. p53 ${ }^{\mathrm{Q} /+}+$ TAM group with $n=8$, $\mathrm{p} 53^{\mathrm{Q} / \mathrm{fl}}+$ TAM group with $\mathrm{n}=13$. Mean \pm SEM, Student's t-test. ns, not significant.

(F) Representative immunofluorescence of p53 for TAM-treated p53 ${ }^{\mathrm{Q} /+}$ ('no LOH') and TAMtreated $\mathrm{p} 53^{\mathrm{Q} / \mathrm{fl}}$ mice ('LOH') at endpoint 6 wks post-TAM. Scale bars, $100 \mu \mathrm{m}$.

(G) Total number of invasive tumors (left) and numbers of mice with non-invasive and invasive tumors and (right) of oil-treated $\mathrm{p} 53^{\mathrm{Q} / \mathrm{fl}}$ mice and TAM-treated $\mathrm{p} 53^{\mathrm{Q} /+}$ mice (combined as 'no LOH' group) versus TAM-treated $p 53^{\mathrm{Q} / \mathrm{fl}}$ mice ('LOH' group) analyzed at 6-8 wks post oil/TAM. (left) 'no LOH', $\mathrm{n}=27$ tumors from 7 mice analyzed and 'LOH', $\mathrm{n}=49$ tumors from 8 mice analyzed. (right) 'no LOH' $\mathrm{n}=7$ mice and 'LOH' $\mathrm{n}=8$ mice. Fisher's exact test. Bars, mean \pm SEM. $p^{* * *} \leq 0.001$.

(H) Representative histopathology of two LOH p53 $3^{\mathrm{Q} / \mathrm{fl}}$ tumors 8 wks after TAM treatment. LOH induction showing (left) extensive invasion deep within the muscularis propria of the bowel wall (black arrow) and the muscularis mucosae (white arrows). The deeply invasive malignant gland 
418 (black arrow) is ruptured, spilling its content into mucous lakes with single tumor cells floating in it (\#). Right, invading glands in the submucosa $\left(^{*}\right)$. H\&E, scale bars $100 \mu \mathrm{m}$.

420

421

422

423

424

425

426

427

428

429

430

431

432

433

434

435

436

437

438

439

440

441

442

443

444

445

446

447

448

449

450

451

452

(I) Representative immunofluorescence of two invasive tumors stabilized for mutp53 from TAMtreated p53 ${ }^{\mathrm{Q} / \mathrm{fl}}$ mice ('LOH' group) for p53 (red), a-SMA (smooth muscle marker, green) and DAPI (blue) at endpoint 6 wks. Scale bars, $100 \mu \mathrm{m}$. MM, muscularis mucosae, MP, muscularis propia. White arrows, tumor cells invading the MM. Asterix, small invasive cell clumps invading the submucosa. Note that tips of invading cells are predominantly positive for mutp53.

\section{Supplemental Figure 1. p53 loss-of-heterozygosity is a prerequisite for mutp53 protein stabilization and enables invasion in colorectal cancer}

(A) The humanized GOF TP53 ${ }^{\mathrm{R} 248 \mathrm{Q}}$ allele $\left(\mathrm{p} 53^{\mathrm{Q}}\right.$ ) was paired with the p53null allele ${ }^{85}$ in the AOM/DSS colorectal cancer model previously described in Schulz-Heddergott et al. ${ }^{9}$ to generate heterozygous $\mathrm{p} 53^{\mathrm{Q} /+}$ mice (mimicking no $\mathrm{LOH}$ ) and GOF $\mathrm{p} 53^{\mathrm{Q} /-}$ mice (mimicking $\mathrm{p} 53 \mathrm{LOH}$ ), with corresponding controls ( $\mathrm{p} 53^{-/+}$and $\mathrm{p} 53^{-/-}$mice). All mice were treated with $1.5 \%$ DSS. Time line for p53-proficient (containing one WTp53 allele) and p53-deficient (both p53 alleles are altered) mice used in this study. Endpoint analysis at 10 wks for all mice to avoid losing p53-deficient mice due to lymphoma and intestinal obstruction.

(B) Representative immunofluorescence staining for p53 (green) and DAPI (blue) of CRC tumors from the indicated genotypes at endpoint 10 wks. Occasional $p 53^{\mathrm{Q} /+}$ tumors show a minor focus of stabilized mutp53, presumably an area that underwent p53LOH. Scale bars, 100 $\mu \mathrm{m}$. White arrowheads show invasive malignant glands.

(C) Total tumor numbers per mouse of the indicated genotypes at endpoints described in (A). $\mathrm{p} 53^{-/+}$and $\mathrm{p} 53^{\mathrm{Q} /+}$ mice harbor heterozygous CRC tumors. Tumors from $\mathrm{p} 53^{-/-}$and $\mathrm{p} 53^{\mathrm{Q} /-}$ mice are homozygous for their TP53 alteration mimicking p53LOH. Bars indicate mean \pm SEM, Student's t-test. $\mathrm{p}^{*}=0.05 ; \mathrm{p}^{* * *}=0.001$; ns, not significant.

(D) Total number of mice with non-invasive and invasive tumors (left) and total number of invasive tumors (right) of the indicated genotypes from $(A-C)$ at endpoints described in (A). (left) $\mathrm{p} 53^{-/+}$and $\mathrm{p} 53^{\mathrm{Q} /+}, \mathrm{n}=7$ mice each. $\mathrm{p} 53^{-/-}$and $\mathrm{p} 53^{\mathrm{Q} /-}, \mathrm{n}=16$ mice each. (right) $\mathrm{p} 53^{-/+}, \mathrm{n}=42$ tumors from 7 mice; $\mathrm{p} 53^{\mathrm{Q} /+}, \mathrm{n}=45$ tumors from 7 mice; $\mathrm{p} 53^{-{ }^{--}}, \mathrm{n}=71$ tumors from 16 mice and $\mathrm{p} 53^{\mathrm{Q} /-}, \mathrm{n}=115$ tumors from 16 mice. Bars, mean \pm SEM. Fisher's exact test. $\mathrm{p}^{\star * *} \leq 0.05, \mathrm{p}^{* *} \leq$ 0.01 .

(E) Representative histopathology of $\mathrm{p} 53^{\mathrm{Q} /-}$ tumors. H\&E staining, Scale bars, $100 \mu \mathrm{m}$. Dashed line, muscularis mucosae; dashed/dot line, border to muscularis propra. 
Figure 2. The WTp53 allele in heterozygous colorectal tumors retains its activity and represses HSF1 target gene expression in vivo

455

456

457

458

459

460

461

462

463

464

465

466

467

468

469

470

471

472

473

474

475

476

477

478

479

480

481

482

483

484

485

486

(A) Mdm2 mRNA levels of untreated CRC tumors from the indicated genotypes. Single colonic tumors from the indicated genotypes were pooled ( $\geq 5$ tumors per group). qRT-PCR normalized to $36 B 4$ mRNA. Mean \pm SEM of 3 technical replicates, each in triplicates. Student's t-test.

(B) Scheme of Nutlin treatment in the AOM/DSS colorectal tumor model. After AOM/DSS induction, tumor growth was quantitated by serial colonoscopy ('colos'). Mice with the defined tumor burden of at least 2-3 S2 tumors and at least one S3 tumor were orally treated with vehicle or $150 \mathrm{mg} / \mathrm{kg}$ Nutlin for 3 days. Tumors were analyzed $8 \mathrm{hrs}$ after the last treatment.

(C, D) mRNA levels of WTp53 target genes (C) or HSF1 target genes (D) of colonic p53 ${ }^{\mathrm{Q} /+}$ and

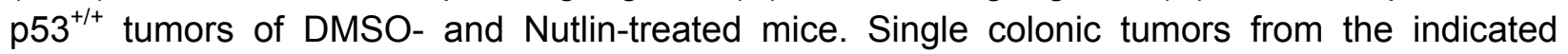
genotypes were pooled ( $\geq 5$ tumors per group). qRT-PCR normalized to 36B4 mRNA. Mean \pm SEM of 3 technical replicates, each in triplicates. Student's t-test.

\section{Supplemental Figure 2. p53 deletion alone is not sufficient to activate HSF1 in vivo}

(A) Scheme and time line of the AOM/DSS colorectal cancer model using p53null mice ${ }^{85}$. Mice were treated as indicated. Endpoint analysis at 12 wks for p53-proficient mice to avoid losing them to extraneous reasons such as intestinal obstruction and anal prolapse. Endpoint analysis at 10 wks for p53-deficient mice to avoid losing them to lymphoma.

(B) Total number of colonic tumors per mouse (left) and tumor size distribution (right) of the indicated genotypes from $(A)$. $n$, total mouse numbers. Bars, mean $\pm S E M$, Student's $t$-test. $p^{* *}=0.01 ; n s$, not significant.

(C) Representative colonoscopy of $\mathrm{p} 53^{+/+}$and $\mathrm{p} 53^{-/-}$mice at endpoint 10 wks post AOM/DSS. White lines outline tumors. Black arrow indicates an S2 tumor and striped arrows indicate S3 tumors. Tumor scoring was performed according to Becker \& Neurath ${ }^{50}$.

(D) Colon sections from 'Swiss roles' of AOM/DSS-treated $\mathrm{p} 53^{+/+}$and $\mathrm{p} 53^{-/-}$mice. H\&E.

(E-G) mRNA levels of cell cycle genes (E), wildtype p53 target genes (F) and HSF1 target genes $(G)$ isolated from the indicated genotypes of colonic tumors (pooled samples, $n \geq 5$ tumors per genotype). qRT-PCR normalized to 36B4 or HPRT mRNA. Mean \pm SEM of 3 technical replicates, in triplicates. Student's t-test. 
(A) Luciferase reporter assay for heat-shock response elements (HSE). HCT116 and RKO cells were co-transfected with HSE-Luc and Renilla plasmids (pRL-TK). $48 \mathrm{hrs}$ later cells were treated with DMSO or $10 \mu \mathrm{M}$ Nutlin for $24 \mathrm{hrs}$. Firefly expression was normalized to Renilla expression and relative light units (RLU) were calculated. Mean \pm SEM of 3 independent experiments, each in triplicates. Student's t test.

(B) HSE Luciferase assay as in (A) upon depletion of WTp53 by shRNA. Control, scramble shRNA. $48 \mathrm{hrs}$ post transfection, cells were treated +/- Nutlin $(10 \mu \mathrm{M})$ for $24 \mathrm{hrs}$. HSF1 binding to its HSE-promoter was measured as in (A). Mean \pm SEM of 3 independent experiments, each in triplicates. Student's t test.

(C) Chaperone-dependent and -independent HSF1 target gene expression in HCT116 and RKO cells treated with DMSO or $10 \mu \mathrm{M}$ Nutlin for $24 \mathrm{hrs}$. qRT-PCR for the indicated mRNAs, each normalized to 36B4 mRNA. Relative values are given in [ratio $\left(2^{- \text {ddCT }}\right)$ ]. Mean $\pm S E M$ of 2 independent experiments, each repeated twice in triplicates. Student's t-test, $p^{*}=0.05, p^{* *}=0.01$, $p^{* * *}=0.001 ; n s$, not significant.

(D) HSF1 target gene expression in RKO cells upon depletion of WTp53. 48 hrs post transfection with two sip53 RNAs or scrambled control siRNA (scr2), RKO cells were treated +/Nutlin $(10 \mu \mathrm{M})$ for $24 \mathrm{hrs}$. qRT-PCR for the indicated mRNAs as in (C). Mean \pm SEM of 2 independent experiments, each repeated twice in triplicates. Student's t-test, $p^{*}=0.05, p^{* *}=0.01$, $506 \quad \mathrm{p}^{* * *}=0.001$.

507 (E) Activated WTp53 suppresses pSer326-HSF1, the key marker of HSF1 activity. HCT116 and 508 RKO cells were treated $+/-$ Nutlin $(10 \mu \mathrm{M})$ for the indicated times. Immunoblot analysis. p53 accumulation indicates p53 activation. Actin, loading control.

(F) Repression of HSF1 target genes (Hsp90 $\alpha$ and Hsp27) and destabilization of the Hsp90 $\alpha$ client proteins AKT, c-Raf and Bcl-xl after p53 activation. HCT116 cells were treated with DMSO or $10 \mu \mathrm{M}$ Nutlin for the indicated times. Immunoblot analysis. Actin, loading control.

(G) Stably HSF1-overexpressing HCT116 subclones (HSF1c1 and HSF1c2) or empty vector control cells (ORF) were treated with DMSO or Nutlin for $24 \mathrm{hrs}$. Representative immunoblot analysis. pSer326-HSF1 shown with short and long exposure times. Actin, loading control.

(H) Nutlin represses HSF1 activity in heat-shocked cells, rescued by p53 knockdown. HSE luciferase assay. HCT116 and RKO cells were transfected with HSE-LUC and Renilla plasmids and shp53 as in (B). $48 \mathrm{hrs}$ post transfection, cells were treated with DMSO or $10 \mu \mathrm{M}$ Nutlin for $24 \mathrm{hrs}$. During the final $2 \mathrm{hrs}$, HCT116 cells were heat-shocked for $1 \mathrm{hr}$ at $42^{\circ} \mathrm{C}$ followed by recovery for $1 \mathrm{hr}$. HSF1 binding to HSE-Luc reporter was measured as in (A). Mean \pm SEM of 3 independent experiments, each in triplicates. Student's t-test, $p^{*}=0.05$; ns, not significant. scrambled (scr2). $48 \mathrm{hrs}$ post transfection, cells were treated with DMSO or $10 \mu \mathrm{M}$ Nutlin for 24 
hrs. During the final $2 \mathrm{hrs}$, HCT116 cells were heat-shocked for $1 \mathrm{hr}$ at $42^{\circ} \mathrm{C}$ followed by recovery for $1 \mathrm{hr}$ as in $(\mathrm{H})$. Immunoblot analysis for pSer326-HSF1. Actin, loading control.

\section{Supplemental Figure 3. HSF1 activity is repressed by WTp53 in human colorectal cancer cells}

(A) p53-induced HSF1 target gene repression is rescued by WTp53 silencing. HCT116 cells were transfected with siRNAs for p53 or scrambled control siRNA (scr2) for $48 \mathrm{hrs}$. Cells were treated with DMSO or $10 \mu \mathrm{M}$ Nutlin for $24 \mathrm{hrs}$. qRT-PCRs for the indicated mRNAs, each normalized to 36B4 mRNA. Relative values are given in [ratio $\left(2^{- \text {ddCT }}\right)$ ]. Mean \pm SEM of 2 independent experiments, each repeated in triplicates. Student's t-test, $p^{*}=0.05, p^{* *}=0.01$, $\mathrm{p}^{* * *}=0.001 ; \mathrm{ns}$, not significant.

(B) WTp53 harboring LS513 and LS174T cells were treated with DMSO or $10 \mu \mathrm{M}$ Nutlin for the indicated times. Representative immunoblot analysis for pSer326-HSF1, the key marker of HSF1 activity. Actin, loading control.

(C) p53 silencing abrogates HSF1 inactivation upon Nutlin. HCT116 cells were transfected with two different siRNAs against p53 or scrambled control siRNA (scr). 48 hrs post-transfection, cells were treated with DMSO or $10 \mu \mathrm{M}$ Nutlin for $24 \mathrm{hrs}$. Cell lysates were immunoblotted for pSer326-HSF1, total HSF1 (tHSF1) and p53. Actin, loading control.

(D) p53 deletion prevents Nutlin-induced HSF1 inactivation. Isogenic HCT116 cells (p53 ${ }^{+/+}$vs p53-/-, harboring a p53 Exon2 deletion) were left untreated (un) or treated with DMSO or Nutlin for $24 \mathrm{hrs}$. Representative immunoblots for pSer326-HSF1 and p53. Actin, loading control.

(E) mutp53-containing CRC cells failed to reduce pSer326-HSF1 after Nutlin. SW480 cells treated + /- Nutlin $(20 \mu \mathrm{M})$ for the indicated hours. Representative immunoblot. Actin, loading control.

(F) Stably HSF1-overexpressing HCT116 subclone HSF1c1 and its empty vector control line (ORF) were treated with DMSO or $10 \mu \mathrm{M}$ Nutlin for $24 \mathrm{hrs}$. qRT-PCR analysis of the indicated HSF1 target genes. Mean \pm SEM of 2 independent experiments, each repeated twice in triplicates. Student's t-test, $p^{*}=0.05, p^{* *}=0.01 ; n s$. 
Figure 4. p53 suppresses HSF1 activity via cyclin-dependent kinase inhibitor CDKN1A/p21 in human CRC cells

(A) p21 silencing attenuates p53-induced HSF1 inactivation. RKO cells were transfected with different siRNAs against p21 and p53 or scrambled control siRNA (scr2) for 48 hrs. Cells were then treated with DMSO or $10 \mu \mathrm{M}$ Nutlin for $24 \mathrm{hrs}$. Representative Immunoblot analysis for pSer326-HSF1, p21 and p53. Actin, loading control.

(B) Rescue of p53-induced HSF1 target gene suppression by depletion of p21 in RKO cells. 48 hrs post transfection with siRNAs against p21 or scrambled control siRNA (scr2), cells were treated with DMSO or $10 \mu \mathrm{M}$ Nutlin for $24 \mathrm{hrs}$. qRT-PCR of the indicated mRNAs, normalized to 36B4 mRNA. Relative values given in [ratio $\left(2^{-\mathrm{ddCT}}\right)$ ]. Mean $\pm S E M$ of 2 independent experiments, each repeated twice in triplicates. Student's t-test, $\mathrm{p}^{*}=0.05$; ns, not significant.

(C) WTp53 harboring CRC cell lines were treated with DMSO, $10 \mu \mathrm{M}$ Palbociclib (Palbo) or 10 $\mu \mathrm{M}$ Nutlin for $24 \mathrm{hrs}$. Cell cycle inhibition was confirmed by Rb de(hypo)phosphorylation. Immunoblot analysis for the indicated proteins. pRb, phospho-Rb. Actin, loading control.

(D) HSF1 target gene repression after direct cell cycle inhibition. RKO cells were treated with 10 $\mu \mathrm{M}$ Palbociclib (CDK4/6 inhibitor), $10 \mu \mathrm{M}$ Nutlin (MDM2 inhibitor) or DMSO for 24 hrs. qRTPCRs analysis for the indicated mRNAs. Relative values calculated as in (B). Mean $\pm S E M$ of 2 independent experiments, each repeated twice in triplicates. Student's t-test, $p^{*}=0.05, p^{* *}=0.01$, $\mathrm{p}^{* * *}=0.001 ; \mathrm{ns}$, not significant.

(E, F) Cell cycle inhibition by p53 inactivates HSF1 activity. HCT116 cells were treated with DMSO, $10 \mu \mathrm{M}$ Nutlin, $10 \mu \mathrm{M}$ Palbociclib, RG7112 (E) or RG7388/Idasanutlin (F) as indicated for $24 \mathrm{hrs}$. Immunoblot analysis. Actin, loading control.

(G) Cell cycle inhibition prevents pSer326-HSF1 activation in stably HSF1-overexpressing HCT116 cells. HSF1c1 or empty vector control (ORF) cells were treated with DMSO, $\mathrm{H}_{2} \mathrm{O}, 10$ $\mu \mathrm{M}$ Nutlin or $10 \mu \mathrm{M}$ Palbociclib for 24 hrs. Representative immunoblot analysis. Actin, loading control.

(H) Cell cycle inhibition in HSF1-overexpressing HSF1c1 cells strongly repress HSF1 target gene expression. HSF1c1 or ORF control cells were exposed to $\mathrm{H}_{2} \mathrm{O}$ or Palbociclib (10 $\left.\mu \mathrm{M}\right)$ for $24 \mathrm{hrs}$. qRT-PCR analysis of the indicated HSF1 target genes. Mean \pm SEM of 2 independent experiments, each repeated twice in triplicates. Student's t-test, $p^{*}=0.05, p^{* *}=0.01, p^{* * *}=0.001$; ns, not significant.

(I) Direct CDK4/6 inhibition drives HSF1 inactivation. HCT116 cells were treated with DMSO, 10 $\mu \mathrm{M}$ Nutlin, $10 \mu \mathrm{M}$ Palbociclib, $0.5 \mu \mathrm{M}$ and $10 \mu \mathrm{M}$ RO3306 and $20 \mu \mathrm{M}$ Roscovitine (Rosco) for 24 hrs. Representative immunoblot. Actin, loading control. 
591 Supplemental Figure 4. p53 suppresses HSF1 activity via cyclin-dependent kinase 592 inhibitor CDKN1A/p21 in human CRC cells

593 (A) Analysis of CDKN1A/p21 mRNA expression. One representative qRT-PCR each shown for 594 HCT116 and RKO cells. Cells were transfected with siRNAs against CDKN1A/p21 and p53 or 595 scrambled control siRNA (scr2) for $48 \mathrm{hrs,} \mathrm{followed} \mathrm{by} \mathrm{DMSO} \mathrm{or} 10 \mu \mathrm{M}$ Nutlin treatment for 24 596 hrs. Relative values of CDKN1A mRNA normalized to 36B4 mRNA and given in [ratio $\left(2^{\text {-ddCT }}\right)$ ].

597 (B) Analysis of HSF1 target gene expression in HCT116 cells upon depletion of p21. 48 hrs post 598 transfection with two siRNAs against p21 or scrambled control siRNA (scr2), HCT116 cells were 599 treated with DMSO or $10 \mu \mathrm{M}$ Nutlin for $24 \mathrm{hrs}$. qRT-PCR analysis. Mean \pm SEM of 2 600 independent experiments, each repeated twice in triplicates. Relative values were calculated as 601 in (A). Student's t-test, $\mathrm{p}^{*}=0.05, \mathrm{p}^{* *}=0.01, \mathrm{p}^{* * *}=0.001 ; \mathrm{ns}$, not significant. 
Figure 5. WTp53 activation represses MLK3. MLK3 links cell cycle to the MAPK stress pathway to activate the HSF1 response

(A) Expression of cell cycle progression genes is inhibited by p53 activation. HCT116 cells were transfected with siRNAs for p53 or scrambled control siRNA (scr2) for 48 hrs, followed by DMSO or $10 \mu \mathrm{M}$ Nutlin treatment for $24 \mathrm{hrs}$. qRT-PCRs for the indicated mRNAs, each normalized to 36B4 mRNA. MLK3, Mixed lineage kinase 3. PLK4, polo-like kinas 4. Relative values as ratio $\left(2^{-\mathrm{ddCT}}\right)$. Mean $\pm S E M$ of 2 independent experiments, each repeated in triplicates. Student's t-test, $\mathrm{p}^{*}=0.05, \mathrm{p}^{* *}=0.01, \mathrm{p}^{* * *}=0.001$.

(B) MLK3 silencing suppresses pSer326-HSF1, mimicking that seen by p53 activation. Indicated cells were transfected with an siRNAs pool against MLK3 or scrambled control siRNA (scr2). 48 hrs post-transfection, cells were treated with DMSO or $10 \mu \mathrm{M}$ Nutlin for $24 \mathrm{hrs}$. Immunoblot analysis. GAPDH, loading control.

(C) MLK3 silencing abrogates HSF1 target gene expression, mimicking that seen by p53 activation. HCT116 cells were transfected and treated as in (B). qRT-PCRs for the indicated mRNAs, each normalized to 36B4 mRNA. Relative values were calculated as in $(A)$. Mean \pm SEM of 2 independent experiments, each repeated in triplicates. Student's t-test, $p^{*}=0.05$, $\mathrm{p}^{* *}=0.01, \mathrm{p}^{* * *}=0.001$.

(D) HSF1 target gene expression is attenuated after MLK3 depletion. Indicated cells were transfected with an siRNAs pool against MLK3 or scrambled control siRNA (scr2). 72 hrs posttransfection, qRT-PCRs for the indicated mRNAs was performed. Normalized to 36B4 mRNA. Relative values and means as in (A). Mean \pm SEM of 2 independent experiments, each repeated in triplicates. Student's t-test, $\mathrm{p}^{*}=0.05, \mathrm{p}^{* *}=0.01, \mathrm{p}^{* * *}=0.001$.

(E-G) Cell cycle inhibition reduces MLK3 expression and causes MEK1 inactivation. The indicated cells were treated for $24 \mathrm{hrs}$ with DMSO, $10 \mu \mathrm{M}$ Nutlin or $10 \mu \mathrm{M}$ Palbociclib (CDK4i) $(E, G), R G 7112(F)$ and RO3306 (G) at the indicated concentrations. Immunoblot analysis. GAPDH, loading control.

\section{Supplemental Figure 5. Cell cycle aberrations activate the MEK pathway and regulate HSF1 activity}

(A, B) Depletion of CDK1 (A) and CDK2 (B) fail to abrogate HSF1 activity. The indicated cells were transfected with two different siRNAs each. 72 hrs post-transfection, cell lysates were analyzed by immunoblots. Actin, loading control.

C) PLK4 silencing in RKO cells from (D). Cells were transfected with 2 different siRNAs against PLK4 or scrambled control siRNA (scr) for 72 hrs. qRT-PCRs for PLK4 mRNAs normalized to 36B4 mRNA. Relative values, ratio $\left(2^{\text {-ddCT }}\right)$. Mean \pm SEM of 2 independent experiments, each repeated in triplicates. Student's t-test, $p^{*}=0.05, p^{* *}=0.01, p^{* * *}=0.001$. 
639 (D) Despite PLK4 mRNA silencing (C), PLK4 protein and pSer326-HSF1 levels are stable, 640 excluding PLK4 as HSF1-activating kinase. Immunoblot analysis of RKO cells from (C).

641 (E) Activated WTp53 strongly reduces MLK3 protein levels. The indicated CRC cells were 642 treated with DMSO or $10 \mu \mathrm{M}$ Nutlin for 36 hrs. Immunoblot analysis. Actin, loading control.

643

644 
645

646

647

648

649

650

651

652

653

654

655

656

657

658

659

660

661

662

663

664

665

666

667

668

669

670

671

672

673

674

675

676

677

678

679

680

681

682

683

Figure 6. p53LOH combined with p53 missense mutations shortens patient survival and upregulates HSF1 activity in human colorectal cancer

(A) Proportion of human colorectal adenocarcinoma samples with $\mathrm{p} 53 \mathrm{LOH}$ versus no-p53LOH. Analysis of the latest version of the COADREAD TCGA dataset, grouped for all types of TP53 mutations (MS, missense; FS, frameshift; NS, nonsense) versus TP53 missense mutations-only (MS). p53LOH samples (= shallow deletion) were determined by TP53 copy number alterations. MS/FS/NS column, p53LOH samples in red of $n=222$ patients, and no-p53LOH in blue of $n=57$ patients. MS column, p53LOH samples in red with $n=168$ patients, and no-p53LOH in blue with $n=43$.

(B) Kaplan-Meier survival curve of all available patients from COADREAD TCGA database. Colorectal cancer patients harboring homozygous WT TP53 were compared to patients harboring missense (MS) p53 mutations plus p53LOH (= shallow deletions). The mean survival of WTp53 patients $(n=214)$ is 83.2 month versus 57.2 months for patients with MS plus p53LOH (mutp53/-) $(n=166)$. Kaplan-Meier statistic on patient cohorts from TCGA, log-rank test, $p=0.19$. Note that TCGA data contains insufficient numbers of heterozygous patients (mutp53/+), precluding statistical analysis.

(C) Heatmap of HSF1 target genes analyzed from colorectal adenocarcinoma patients (COADREAD cohort TCGA database). Patients harboring homozygous TP53 ${ }^{+/+}$(WT TP53) were compared to patients harboring TP53 missense (MS) mutations plus p53LOH (= shallow deletions) $\left(\mathrm{TP} 53^{\mathrm{MS}}+\mathrm{LOH}\right)$. Patient numbers are indicated. Genes were ordered from top to bottom by their relative upregulation (red) and downregulation (blue) and their p-value significance in t-tests. The HSF1 target gene panel from Mendillo et al was used ${ }^{39}$. Note, HSF1 negatively regulates a subset of target genes.

\section{Supplemental Figure 6. p53LOH combined with p53 missense mutations upregulates} HSF1 activity in colorectal and breast cancer patients

(A) Heatmap of HSF1 target genes analyzed from colorectal adenocarcinoma patients (COADREAD cohort TCGA database). Patients harboring homozygous TP53 ${ }^{+/+}$(WT TP53) were compared to patients harboring TP53 alterations (MS, missense; FS, frameshift; NS, nonsense) plus p53LOH (= shallow deletions) $\left(\mathrm{TP} 53^{\mathrm{MS} / \mathrm{FS} / \mathrm{NS}}+\mathrm{LOH}^{\mathrm{L}}\right)$. Patient numbers are indicated. (A-C) Genes were ordered from top to bottom by their relative upregulation (red) and downregulation (blue) and their p-value significance in t-tests. The HSF1 target gene panel from Mendillo et al was used ${ }^{39}$. Note, HSF1 negatively regulates a subset of target genes.

(B, C) Heatmap of HSF1 target genes analyzed from breast cancer patients (BRCA cohort TCGA database). Patients harboring homozygous TP53 ${ }^{+/}$(WT TP53) were compared to patients harboring all TP53 alterations (MS, missense; FS, frameshift; NS, nonsense) plus p53LOH (= shallow deletions) (TP53 $\left.{ }^{\mathrm{MS} / \mathrm{FS} / \mathrm{NS}}+\mathrm{LOH}\right)$ in $(\mathrm{B})$, and also compared to patients harboring TP53 missense (MS) mutations-only plus p53LOH (= shallow deletions) (TP53 ${ }^{\mathrm{MS}+\mathrm{LOH}}$ ) in (C). Patient numbers are indicated. 
684 (D) The MCF7 breast cancer cell line harboring homozygous WTp53 and the MDA-MB-231 685 breast cancer cell line harboring homozygous mutp53 R280K missense mutation were treated 686 with DMSO, Nutlin or Idasanutlin (RG7388) for 24 hrs as indicated. Representative immunoblot 687 analysis. Actin, loading control.

688

689 
Figure 7. In murine CRC organoids p53LOH enables HSF1 activity and triggers mutp53 stabilization

(A) Scheme for treatment of colonic tumor-derived organoids. Heterozygous p53 ${ }^{\mathrm{Q} / \mathrm{fl}}$; vilCreER ${ }^{\mathrm{T} 2}$ mice were treated with AOM/DSS and tumor burden was visualized via colonoscopy. Tumors arisen between 6-8 wks post AOM were resected and processed for colonic organoid cultures. p53LOH was induced by adding $4 \mathrm{OHT}$ (4OH-Tamoxifen) for $24 \mathrm{hrs}$ to activate the CreER ${ }^{\mathrm{T} 2}$ recombinase and create $\mathrm{p} 53^{\mathrm{Q} / \Delta}$ organoids. $\mathrm{EtOH}$, control treatment (no-LOH, p53 ${ }^{\mathrm{Q} / \mathrm{fl}}$ ). Two days after p53LOH induction, organoids were treated with $10 \mu \mathrm{M}$ Nutlin or DMSO for $24 \mathrm{hrs}$ and harvested for analysis.

(B, C) mRNA levels of p53 target genes (B) and HSF1 target genes (C) isolated from colonic p53 ${ }^{\mathrm{Q} / \mathrm{fl}}$ organoids treated as indicated. qRT-PCR normalized to HPRT mRNA. Mean \pm SEM of 3 different organoid cultures (generated from 3 different mice) each measured in triplicates. Student's t-test, $\mathrm{p}^{*}=0.05, \mathrm{p}^{* *}=0.01, \mathrm{p}^{* * *}=0.001 ; \mathrm{ns}$, not significant.

(D) Quantification of (E) using a score for colonic organoids exhibiting nuclear p53 staining. Each dot indicates one organoid of the indicated treatment groups. Nuclear p53 staining score: $0=$ no positive nucleus per organoid; $1=1-20 \%$ positive nuclei per organoid; $2=20-50 \%$ positive nuclei per organoid and $3>50 \%$ positive nuclei per organoid.

(E) Representative immunofluorescence staining of the indicated p53 ${ }^{\mathrm{Q} / \mathrm{fl}}$ organoid groups for $\mathrm{p} 53$ (red), E-cadherin (Ecad, green) and DAPI (blue). Scale bars, $100 \mu \mathrm{m}$.

(F) mRNA levels of pre-miR34a, Vim and Snai1 of colonic $\mathrm{p} 53^{\mathrm{Q} / \mathrm{fl}}$ organoids treated as indicated. qRT-PCR normalized to HPRT mRNA. Mean \pm SEM of 3 different organoid cultures (generated from 3 different mice) each measured in triplicates. Student's t-test, $p^{* *}=0.01, p^{* * *}=0.001$; ns, not significant.

\section{Supplemental Figure 7. Analysis of heterozygous CRC organoids}

(A) Scheme for treatment of colonic tumor-derived organoids. Heterozygous p53 ${ }^{\mathrm{Q} / \mathrm{fl}}$; vilCreER ${ }^{\mathrm{T} 2}$ mice were treated with AOM/DSS and tumor burden was visualized via colonoscopy. Tumors arisen between 6-8 wks post AOM were resected and processed for colonic organoid cultures. p53LOH was induced by adding $4 \mathrm{OHT}\left(4 \mathrm{OH}-\right.$ Tamoxifen) for $24 \mathrm{hrs}$ to activate the CreER ${ }^{\mathrm{T} 2}$ recombinase and create $\mathrm{p} 53^{\mathrm{Q} / \Delta}$ organoids. EtOH, control treatment (no- $\mathrm{LOH}, \mathrm{p} 53^{\mathrm{Q} / \mathrm{fl}}$ ). Two days after p53LOH induction, organoids were treated with $10 \mu \mathrm{M}$ Nutlin or DMSO for 24 hrs and harvested for analysis.

(B) The heterozygous p53 genotype in CRC organoids is stable. Two randomly chosen organoid cultures (generated from 2 different heterozygous TP53 ${ }^{\mathrm{R} 248 \mathrm{Q} / \mathrm{fl}}$; vilCreER ${ }^{\mathrm{T} 2}$ mice) were followed during p2-p7 passaging in vitro. The p53 floxed allele and the $p 53^{\mathrm{Q}}$ allele are indicated. 
726 (C) Incomplete recombination. Trp53 mRNA levels isolated from colonic organoids after 727 p53LOH induction by 4 OHT treatment. qRT-PCR normalized to HPRT mRNA. Mean \pm SEM of 3 728 different organoid cultures generated from 3 different mice each measured in triplicates. The 729 dotted line indicates the value corresponding to 1 copy of the TP53 gene. 
732 1. Fearon, E.R. \& Vogelstein, B. A genetic model for colorectal tumorigenesis. Cell 61, 759-767 (1990).

2. Levine, A.J. \& Oren, M. The first 30 years of p53: growing ever more complex. Nat Rev Cancer $\mathbf{9}$, 749-758 (2009).

736

737

3. Walerych, D., Lisek, K. \& Del Sal, G. Mutant p53: One, No One, and One Hundred Thousand. Front Oncol 5, 289 (2015).

738

739

740

4. Brosh, R. \& Rotter, V. When mutants gain new powers: news from the mutant p53 field. Nat Rev

\section{Cancer 9, 701-713 (2009).}

5. Bykov, V.J.N., Eriksson, S.E., Bianchi, J. \& Wiman, K.G. Targeting mutant p53 for efficient cancer therapy. Nat Rev Cancer 18, 89-102 (2018).

743 7. Schwitalla, S. et al. Loss of p53 in enterocytes generates an inflammatory microenvironment

8. Cooks, T. et al. Mutant p53 prolongs NF-kappaB activation and promotes chronic inflammation and inflammation-associated colorectal cancer. Cancer Cell 23, 634-646 (2013).

9. Schulz-Heddergott, R. et al. Therapeutic Ablation of Gain-of-Function Mutant p53 in Colorectal Cancer Inhibits Stat3-Mediated Tumor Growth and Invasion. Cancer Cell 34, 298-314 e297 (2018).

10. Cancer Genome Atlas, N. Comprehensive molecular characterization of human colon and rectal cancer. Nature 487, 330-337 (2012).

11. Goldstein, I. et al. Understanding wild-type and mutant p53 activities in human cancer: new landmarks on the way to targeted therapies. Cancer Gene Ther 18, 2-11 (2011).

12. Joerger, A.C. \& Fersht, A.R. Structural biology of the tumor suppressor p53. Annu Rev Biochem 77, 557-582 (2008).

13. Olivier, M., Hollstein, M. \& Hainaut, P. TP53 mutations in human cancers: origins, consequences, and clinical use. Cold Spring Harb Perspect Biol 2, a001008 (2010).

14. Schulz-Heddergott, R. \& Moll, U.M. Gain-of-Function (GOF) Mutant p53 as Actionable Therapeutic Target. Cancers (Basel) 10 (2018).

15. Olive, K.P. et al. Mutant p53 gain of function in two mouse models of Li-Fraumeni syndrome. Cell 119, 847-860 (2004).

16. Terzian, T. et al. The inherent instability of mutant p53 is alleviated by Mdm2 or p16INK4a loss. Genes Dev 22, 1337-1344 (2008).

17. Hanel, W. et al. Two hot spot mutant p53 mouse models display differential gain of function in tumorigenesis. Cell Death Differ 20, 898-909 (2013).

18. Lang, G.A. et al. Gain of function of a p53 hot spot mutation in a mouse model of Li-Fraumeni syndrome. Cell 119, 861-872 (2004).

19. Nakayama, M. et al. Intestinal cancer progression by mutant p53 through the acquisition of invasiveness associated with complex glandular formation. Oncogene 36, 5885-5896 (2017).

20. Stein, Y., Rotter, V. \& Aloni-Grinstein, R. Gain-of-Function Mutant p53: All the Roads Lead to Tumorigenesis. Int J Mol Sci 20 (2019).

21. Freed-Pastor, W.A. \& Prives, C. Mutant p53: one name, many proteins. Genes Dev 26, 12681286 (2012).

22. Kim, M.P. \& Lozano, G. Mutant p53 partners in crime. Cell Death Differ 25, 161-168 (2018).

777

23. Bellazzo, A., Sicari, D., Valentino, E., Del Sal, G. \& Collavin, L. Complexes formed by mutant p53 and their roles in breast cancer. Breast Cancer (Dove Med Press) 10, 101-112 (2018). 
24. Muller, P.A.J. \& Vousden, K.H. Mutant p53 in Cancer: New Functions and Therapeutic Opportunities. Cancer Cell 25, 304-317 (2014).

25. Pfister, N.T. \& Prives, C. Transcriptional Regulation by Wild-Type and Cancer-Related Mutant Forms of p53. Cold Spring Harb Perspect Med 7 (2017).

26. Zhang, Y. et al. Somatic Trp53 mutations differentially drive breast cancer and evolution of metastases. Nat Commun 9, 3953 (2018).

27. Blagosklonny, M.V., Toretsky, J., Bohen, S. \& Neckers, L. Mutant conformation of p53 translated in vitro or in vivo requires functional HSP90. Proc Natl Acad Sci U S A 93, 8379-8383 (1996).

28. Whitesell, L., Sutphin, P.D., Pulcini, E.J., Martinez, J.D. \& Cook, P.H. The physical association of multiple molecular chaperone proteins with mutant p53 is altered by geldanamycin, an hsp90binding agent. Mol Cell Biol 18, 1517-1524 (1998).

29. Muller, P., Hrstka, R., Coomber, D., Lane, D.P. \& Vojtesek, B. Chaperone-dependent stabilization and degradation of p53 mutants. Oncogene 27, 3371-3383 (2008).

30. Ingallina, E. et al. Mechanical cues control mutant p53 stability through a mevalonate-RhoA axis. Nat Cell Biol 20, 28-35 (2018).

31. Lee, M.K. et al. Cell-type, dose, and mutation-type specificity dictate mutant p53 functions in vivo. Cancer Cell 22, 751-764 (2012).

32. Li, D., Marchenko, N.D. \& Moll, U.M. SAHA shows preferential cytotoxicity in mutant p53 cancer cells by destabilizing mutant p53 through inhibition of the HDAC6-Hsp90 chaperone axis. Cell Death Differ 18, 1904-1913 (2011).

33. Li, D. et al. Functional inactivation of endogenous MDM2 and CHIP by HSP90 causes aberrant stabilization of mutant p53 in human cancer cells. Mol Cancer Res 9, 577-588 (2011).

34. Anckar, J. \& Sistonen, L. Regulation of HSF1 function in the heat stress response: implications in aging and disease. Annu Rev Biochem 80, 1089-1115 (2011).

35. Gomez-Pastor, R., Burchfiel, E.T. \& Thiele, D.J. Regulation of heat shock transcription factors and their roles in physiology and disease. Nat Rev Mol Cell Biol 19, 4-19 (2018).

36. Whitesell, L. \& Lindquist, S. Inhibiting the transcription factor HSF1 as an anticancer strategy. Expert Opin Ther Targets 13, 469-478 (2009).

37. Dai, C., Whitesell, L., Rogers, A.B. \& Lindquist, S. Heat shock factor 1 is a powerful multifaceted modifier of carcinogenesis. Cell 130, 1005-1018 (2007).

38. Miyata, Y., Nakamoto, H. \& Neckers, L. The therapeutic target Hsp90 and cancer hallmarks. Curr Pharm Des 19, 347-365 (2013).

39. Mendillo, M.L. et al. HSF1 drives a transcriptional program distinct from heat shock to support highly malignant human cancers. Cell 150, 549-562 (2012).

40. Toma-Jonik, A., Vydra, N., Janus, P. \& Widlak, W. Interplay between HSF1 and p53 signaling pathways in cancer initiation and progression: non-oncogene and oncogene addiction. Cell Oncol (Dordr) 42, 579-589 (2019).

41. Alexandrova, E.M. et al. p53 loss-of-heterozygosity is a necessary prerequisite for mutant p53 stabilization and gain-of-function in vivo. Cell Death Dis 8, e2661 (2017).

42. Hingorani, S.R. et al. Trp53R172H and KrasG12D cooperate to promote chromosomal instability and widely metastatic pancreatic ductal adenocarcinoma in mice. Cancer Cell 7, 469-483 (2005).

43. Baker, S.J. et al. Chromosome 17 deletions and p53 gene mutations in colorectal carcinomas. Science 244, 217-221 (1989).

44. Parikh, N. et al. Effects of TP53 mutational status on gene expression patterns across 10 human cancer types. J Pathol 232, 522-533 (2014).

45. Jackson, E.L. et al. The differential effects of mutant p53 alleles on advanced murine lung cancer. Cancer Res 65, 10280-10288 (2005). 
46. Donehower, L.A. et al. Integrated Analysis of TP53 Gene and Pathway Alterations in The Cancer Genome Atlas. Cell Rep 28, 1370-1384 e1375 (2019).

47. Donehower, L.A. et al. Integrated Analysis of TP53 Gene and Pathway Alterations in The Cancer Genome Atlas. Cell Rep 28, 3010 (2019).

48. Muzumdar, M.D. et al. Clonal dynamics following p53 loss of heterozygosity in Kras-driven cancers. Nat Commun 7, 12685 (2016).

49. Shetzer, Y. et al. The onset of p53 loss of heterozygosity is differentially induced in various stem cell types and may involve the loss of either allele. Cell Death Differ 21, 1419-1431 (2014).

50. Becker, C., Fantini, M.C. \& Neurath, M.F. High resolution colonoscopy in live mice. Nat Protoc 1, 2900-2904 (2006).

51. Ghaleb, A., Yallowitz, A. \& Marchenko, N. Irradiation induces p53 loss of heterozygosity in breast cancer expressing mutant p53. Commun Biol 2, 436 (2019).

52. Li, D., Yallowitz, A., Ozog, L. \& Marchenko, N. A gain-of-function mutant p53-HSF1 feed forward circuit governs adaptation of cancer cells to proteotoxic stress. Cell Death Dis 5, e1194 (2014).

53. Esser, C., Scheffner, M. \& Hohfeld, J. The chaperone-associated ubiquitin ligase CHIP is able to target p53 for proteasomal degradation. J Biol Chem 280, 27443-27448 (2005).

54. Iyer, S.V. et al. Allele-specific silencing of mutant p53 attenuates dominant-negative and gain-offunction activities. Oncotarget 7, 5401-5415 (2016).

55. Kern, S.E. et al. Oncogenic forms of p53 inhibit p53-regulated gene expression. Science 256, 827830 (1992).

56. Sabapathy, K. The Contrived Mutant p53 Oncogene - Beyond Loss of Functions. Front Oncol 5, 276 (2015).

57. Shahbandi, A. \& Jackson, J.G. Analysis across multiple tumor types provides no evidence that mutant p53 exerts dominant negative activity. NPJ Precis Oncol 3, 1 (2019).

58. Schulz, R. et al. HER2/ErbB2 activates HSF1 and thereby controls HSP90 clients including MIF in HER2-overexpressing breast cancer. Cell Death Dis 5, e980 (2014).

59. Polager, S. \& Ginsberg, D. E2F - at the crossroads of life and death. Trends Cell Biol 18, 528-535 (2008).

60. Rattanasinchai, C. \& Gallo, K.A. MLK3 Signaling in Cancer Invasion. Cancers (Basel) 8 (2016).

61. Hartkamp, J., Troppmair, J. \& Rapp, U.R. The JNK/SAPK activator mixed lineage kinase 3 (MLK3) transforms NIH 3T3 cells in a MEK-dependent fashion. Cancer Res 59, 2195-2202 (1999).

62. Schroyer, A.L., Stimes, N.W., Abi Saab, W.F. \& Chadee, D.N. MLK3 phosphorylation by ERK1/2 is required for oxidative stress-induced invasion of colorectal cancer cells. Oncogene 37, 10311040 (2018).

63. Tang, Z. et al. MEK guards proteome stability and inhibits tumor-suppressive amyloidogenesis via HSF1. Cell 160, 729-744 (2015).

64. Vydra, N. et al. 17beta-Estradiol Activates HSF1 via MAPK Signaling in ERalpha-Positive Breast Cancer Cells. Cancers (Basel) 11 (2019).

65. Hanahan, D. \& Weinberg, R.A. Hallmarks of cancer: the next generation. Cell 144, 646-674 (2011).

66. Rokavec, M., Li, H., Jiang, L. \& Hermeking, H. The p53/miR-34 axis in development and disease. J Mol Cell Biol 6, 214-230 (2014).

67. Kimura, A. et al. Nuclear heat shock protein 110 expression is associated with poor prognosis and chemotherapy resistance in gastric cancer. Oncotarget 7, 18415-18423 (2016).

68. Tanaka, T. Development of an inflammation-associated colorectal cancer model and its application for research on carcinogenesis and chemoprevention. Int J Inflam 2012, 658786 (2012). 
908

69. Muller, P.A. et al. Mutant p53 drives invasion by promoting integrin recycling. Cell 139, 13271341 (2009).

70. Wawrzynow, B., Zylicz, A. \& Zylicz, M. Chaperoning the guardian of the genome. The two-faced role of molecular chaperones in p53 tumor suppressor action. Biochim Biophys Acta 1869, 161174 (2018).

71. King, F.W., Wawrzynow, A., Hohfeld, J. \& Zylicz, M. Co-chaperones Bag-1, Hop and Hsp40 regulate $\mathrm{Hsc70}$ and $\mathrm{Hsp90}$ interactions with wild-type or mutant p53. EMBO J 20, 6297-6305 (2001).

72. Boettcher, S. et al. A dominant-negative effect drives selection of TP53 missense mutations in myeloid malignancies. Science 365, 599-604 (2019).

73. Lane, D.P. How to lose tumor suppression. Science 365, 539-540 (2019).

74. Sabapathy, K. \& Lane, D.P. Therapeutic targeting of p53: all mutants are equal, but some mutants are more equal than others. Nat Rev Clin Oncol 15, 13-30 (2018).

75. Weissmueller, S. et al. Mutant p53 drives pancreatic cancer metastasis through cell-autonomous PDGF receptor beta signaling. Cell 157, 382-394 (2014).

76. Sakai, E. et al. Combined Mutation of Apc, Kras, and Tgfbr2 Effectively Drives Metastasis of Intestinal Cancer. Cancer Res 78, 1334-1346 (2018).

77. Bolt, A.B., Papanikolaou, A., Delker, D.A., Wang, Q.S. \& Rosenberg, D.W. Azoxymethane induces $\mathrm{KI}-$ ras activation in the tumor resistant AKR/J mouse colon. Mol Carcinog 27, 210-218 (2000).

78. Takeda, H. et al. Transposon mutagenesis identifies genes and evolutionary forces driving gastrointestinal tract tumor progression. Nat Genet 47, 142-150 (2015).

79. Logan, I.R. et al. Heat shock factor-1 modulates p53 activity in the transcriptional response to DNA damage. Nucleic Acids Res 37, 2962-2973 (2009).

80. Nitta, M., Okamura, H., Aizawa, S. \& Yamaizumi, M. Heat shock induces transient p53dependent cell cycle arrest at G1/S. Oncogene 15, 561-568 (1997).

81. Jin, X., Moskophidis, D., Hu, Y., Phillips, A. \& Mivechi, N.F. Heat shock factor 1 deficiency via its downstream target gene alphaB-crystallin (Hspb5) impairs p53 degradation. J Cell Biochem 107, 504-515 (2009).

82. Muller, L., Schaupp, A., Walerych, D., Wegele, H. \& Buchner, J. Hsp90 regulates the activity of wild type p53 under physiological and elevated temperatures. J Biol Chem 279, 48846-48854 (2004).

83. Walerych, D. et al. Hsp90 chaperones wild-type p53 tumor suppressor protein. J Biol Chem 279, 48836-48845 (2004).

84. Walerych, D. et al. Hsp70 molecular chaperones are required to support p53 tumor suppressor activity under stress conditions. Oncogene 28, 4284-4294 (2009).

85. Jacks, T. et al. Tumor spectrum analysis in p53-mutant mice. Curr Biol 4, 1-7 (1994). 


\section{Online METHODS}

915

916

917

918

919

920

921

922

923

924

925

926

927

928

929

930

931

932

933

934

935

936

937

938

939

940

941

942

943

944

945

946

947

\section{Mouse experiments and genotyping}

Experiments using animal materials were approved by institutional (Göttingen University Medical Center Ethikkommission) and state (Niedersächsisches Landesamt für Verbraucherschutz und Lebensmittelsicherheit, LAVES, Lower Saxony, Germany) committees, ensuring that all experiments conform to the relevant regulatory standards.

The humanized constitutive $T P 53^{R 248 Q}$ (called $p 53^{Q}$ ) knock-in allele has been described in detail $^{1-3}$. Briefly, the human TP53 sequence containing the R248Q mutation in exon 7 replaces part of the mouse Trp53 (exons 4-9). To generate heterozygous mice with one conditional murine Trp53 wildtype allele $\left(p 53^{f l}\right)$, we crossed mice harboring the $p 53^{Q}$ allele with mice harboring the floxed WTp53 allele ${ }^{4}$ flanked by loxP sites in introns 2 and 10 to generate p53 ${ }^{\mathrm{Q} / \mathrm{fl}}$. To remove the floxed WTp53 allele from colonic epithelial tissue, we crossed p53 ${ }^{\mathrm{Q} / \mathrm{fl}}$ mice with villinCreER ${ }^{T 2}$ (called 'ERT2') transgenic mice.

Moreover, the classic Trp53 knock-out mice ( $p 53^{---}$mouse $)^{5}$ were crossed to the $p 53^{Q}$ allele to generate non-tissue specific TP53 alterations (e.g. Supplemental Figure 1, Supplemental Figure 2) as described in Schulz-Heddergott et al. $2018^{3}$.

For all genotypings, we isolated DNA with DirectPCR lysis Reagent (tail) (7Bioscience GmbH). PCR was performed with OneTaq® Quick-Load® 2X Master Mix (New England Biolabs) according to the manufacturer's guidelines using the primers specified in Table S1.

All mouse strains were maintained on a C57BL/6 background for at least 6 generations. For experiments, randomly assigned $10 \mathrm{wk}$ old males and females weighing at least $20 \mathrm{~g}$ were used. Mice were kept under pathogen-free barrier conditions.

\section{Cell culture, treatment and transfection}

Human colorectal cancer cell lines RKO, LS513, LS174T (all harboring WTp53) and SW480 (harboring mutp53 R273H) were cultured in RPMI 1640 medium, isogenic HCT116 WTp53 and HCT115 p53null were cultured in McCoys medium, all supplemented with glutamine, 10\% fetal bovine serum and penicillin/streptomycin and grown in a humidified atmosphere at $37^{\circ} \mathrm{C}$ with $5 \% \mathrm{CO}_{2}$. All cell lines were regularly tested for mycoplasma contamination using the MycoAlert Mycoplasm detection kit (Lonza).

siRNAs were purchased from Ambion/Thermo Fisher Scientific (siRNAs are specified in Table 1) and transfected with Lipofectamine 2000 (Invitrogen). Nutlin-3a (BOC Biosciences), Palbociclib (Sigma), Idasanutlin (RG3788, SelleckChem), RG7112 (SelleckChem), RO-3306 
948

949

950

951

952

953

954

955

956

957

958

959

960

961

962

963

964

965

966

967

968

969

970

971

972

973

974

975

976

977

978

979

980

(Sigma) and Roscovitine (Cell Signaling) were dissolved according to manufacturer's guidelines and used as indicated.

For stable HSF1 expression in HCT116 cells, HEK-293 cells were co-transfected with lentiviral packaging vectors (pMD2.G from Addgene and pCMV-R8.91 from PlasmidFactory Bielefeld) and the Precision LentiORF HSF1 lentiviral plasmid (Id:PLOHS_100008319) or a Precision control plasmid (Dharmacon). After standard lentivirus production, HCT116 cells were transduced in the presence of $8 \mu \mathrm{g} / \mathrm{mL}$ polybrene and cells were selected with Hygromycin for several days. Single cell clones were expanded and validated for HSF1 overexpression by immunostaining with phospho-Ser326 HSF1 (Abcam). Cell clones (HSF1c1, HSF1c2 and ORF control) were cultured in McCoys medium and supplemented as described above.

\section{CRC induction, colonoscopy and treatment}

Murine colorectal carcinoma (CRC) was induced by a single intraperitoneal injection of the colon-selective carcinogen Azoxymethane (AOM, $10 \mathrm{mg} / \mathrm{kg}$ in $0.9 \%$ sodium chloride, Sigma) at the age of 10 wks. After one week rest, an acute colitis was induced with 1.5\% (in p53-deficient mice) or $1.8 \%$ (in p53-proficient mice) dextran sodium sulfate (DSS, MP Biomedicals) for 6 days in the drinking water.

Visualization of tumor growth by mini endoscopy/colonoscopy (Karl Storz GmbH) started 6 wks after AOM induction. Tumor sizes were scored according to the Becker \& Neurath score ${ }^{6}$. Briefly, tumor sizes are calculated relative to the width (luminal circumference) of the colon and scored as sizes 1-5 (S1-S5) with the following specifications: $\mathrm{S} 1=$ just detectable, $\mathrm{S} 2=1 / 8$ of the lumen, $S 3=1 / 4$ of the lumen, $S 4=1 / 2$ of the lumen and $S 5>1 / 2$ of the lumen. Notably, between 6-8 wks post AOM approximately $80 \%$ of mice had at least one S3 tumor and at least three $\mathrm{S} 2$ tumors.

As described in Schulz-Heddergott et al., 2018, for analysis of TP53 ${ }^{R 248 Q}$ mice with either a constitutive p53 wildtype (+) or KO (-) allele, we chose an endpoint type of analysis, ending at 12 wks after AOM in p53-proficient mice (at least one WTp53 allele), or at 10 wks after AOM in all p53-deficient mice (deleted or mutated). This design prevented loss of mice due to colonic obstruction, anal prolapse, or lymphoma development in p53-deficient mice.

For analysis of the inducible p53LOH mouse model we used the TP53 ${ }^{\mathrm{R} 248 \mathrm{Q}}$ allele combined with the conditional floxed WTp53 allele $\left(p 53^{\mathrm{fl}}\right)$ to create heterozygous $\mathrm{p} 53^{\mathrm{Q} / \mathrm{fl}}$; vilCreER ${ }^{\mathrm{T} 2}$ tumors. We specifically induced p53LOH after a defined endoscopy-verified tumor burden was reached (at least one S3 tumor in addition to at least three S2 tumors). After tumor verification, Tamoxifen 
981 (TAM, Sigma) was given by 7 serial intraperitoneal injections ( $1 \mathrm{mg}$ daily per injection in a 1:10 982 ethanol/oil mixture) to activate the inducible recombinase (villinCreER ${ }^{T 2}$ ) and cause p53LOH.

983 Tumor growth was continued to be visualized by colonoscopy over 2 - 8 wks after LOH 984 induction by TAM.

985 At endpoints all mice were euthanized and the entire colon and rectum were harvested. Colons 986 were longitudinally opened, cleaned and displayed. Tumor numbers were counted and tumor 987 sizes measured with a caliper. Tumor biopsies were taken from all mice. To ensure complete 988 sampling of the organ, each colon/rectum was 'swiss rolled', fixed in 4\% paraformaldehyde/PBS 989 and bisected. Both halves were placed face down side-by-side into a single cassette for 990 histologic processing, paraffin embedding and subsequent tissue analysis.

991 Nutlin-3a (BOC Biosciences) treatment was given by oral gavage with $150 \mathrm{mg} / \mathrm{kg}$ per dose over 9923 consecutive days. Mice were sacrificed and colorectal tumors harvested 8 wks after the last 993 treatment.

\section{Histological analysis}

996 Standardized immunohistochemical stainings were performed on murine formalin-fixed paraffin997 embedded (FFPE) tissues. The following primary antibodies were used: p53 FL393 (Santa 998 Cruz, sc-6243), pan-Cytokeratin (Abcam, ab9377) and a-smooth muscle actin/SMA (Abcam, 999 ab21027). The ImmPRESS ${ }^{\text {TM }}$ Peroxidase polymer reagent based on 3, 3-diaminobenzidine 1000 (DAB, Vectorlabs), or Alexa Fluor®488-coupled and Alexa Fluorß647-coupled secondary 1001 antibodies (immunofluorescence) were used as detection systems. Hematoxylin (DAB) or DAPI 1002 (immunofluorescence) were used as counterstains.

1003 To define invasive mouse CRC tumor stages we used the following definition: cancer grown 1004 through the muscularis mucosae into the submucosa (=T1), cancer grown through the 1005 muscularis mucosae and submucosa into the muscularis propria (=T2), cancer grown into the 1006 outermost layers of the colon or rectum and reaching the serosa (=T3). No spread to nearby 1007 lymph nodes or distant metastasis were overserved.

\section{Immunoblots}

1010 Whole cell protein lysates were prepared with RIPA buffer (1\% TritonX-100, 1\% Desoxycholate, $10110.1 \%$ SDS, $150 \mathrm{mM} \mathrm{NaCl}, 10 \mathrm{mM}$ EDTA, 20mM Tris-HCl pH7.5 and complete protease inhibitor 1012 mix, Roche). Tumor tissues were minced and lysed with RIPA buffer followed by sonication. 1013 After centrifugation, protein concentrations were determined by BCA protein assay (Pierce). 1014 Equal amounts of protein lysates were separated by SDS-polyacrylamide gel electrophoresis 
1015

1016

1017

1018

1019

1020

1021

1022

1023

1024

1025

1026

1027

1028

1029

1030

1031

1032

1033

1034

1035

1036

1037

1038

1039

1040

1041

1042

1043

1044

1045

1046

(PAGE), transferred onto nitrocellulose membranes (Millipore), blocked with $5 \%$ milk and probed with the following antibodies: murine p53 (CM5, Vector Laboratories), human p53 (DO1, Santa Cruz sc-126), total HSF1, pMEK1 and CDK1 (all Santa Cruz), HSP90a (Millipore), HSP27, AKT, cRAF, Bcl-XI, CDKN1A/p21, phospho-RB and phospho-S6 (all Cell Signaling), MLK3, phospho-Ser326 HSF1 and CDK2 (all Abcam), PLK4 (Protein Technologies), GAPDH and beta-Actin (both Abcam). Detailed information of antibodies are listed in Table 1.

\section{Quantitative PCR}

Total RNA from cells, tumor tissues or organoids was isolated using the Trizol reagent following manufacturers' guideline (Invitrogen/Thermo Fisher Scientific). Tumor tissues were first homogenized using a homogenizer (T10 basic ULTRA-TURRAX). Equal amounts of RNA were reverse-transcribed (M-MuLV Reverse Transcriptase, NEB), and quantitative real-time PCR (qRT-PCR) analysis was performed using a qPCR Master-Mix (75 mM Tris-HCl pH 8.8, $20 \mathrm{mM}$ $\left(\mathrm{NH}_{4}\right)_{2} \mathrm{SO}_{4}, 0.01 \%$ Tween-20, $3 \mathrm{mM} \mathrm{MgCl}$, SYBR Green 1:80,000, $0.2 \mathrm{mM}$ dNTPs, $20 \mathrm{U} / \mathrm{ml}$ Taq-polymerase, $0.25 \%$ TritonX-100, 300mM Trehalose). Primers are specified in Table S1.

\section{Dual Luciferase Reporter (DLR) Assay}

HSF1 firefly luciferase plasmids harboring seven HSE elements (pGL4.41[/uc2P/HSE/Hygro] vector) and the $\mathrm{pRL}$ (Renilla) luciferase reporter plasmid ( $\mathrm{pRL}-\mathrm{TK}$ ) were purchased from Promega. Cells were seeded and 24 hrs later were co-transfected with $100 \mathrm{ng}$ HSF1Luc plasmids and 200 ng pRL-TK plasmid using Lipofectamine 2000 (Invitrogen). 48 hrs posttransfection, cells were treated with Nutlin as indicated and firefly luciferase and Renilla luciferase activities were measured using a Dual Luciferase Assay. Briefly, cells were lysed with PLB (Passive Lysis Buffer, 5X E194A) and incubated for $15 \mathrm{~min}$. Supernatants were first incubated and measured with firefly luciferase buffer (25 mM Glycylglycine, 15 mM K2HPO4, 4 mM EGTA pH 8.0, $15 \mathrm{mM} \mathrm{MgSO}_{4}, 4$ mM ATP pH 7.0, 1.25 mM DTT, $0.1 \mathrm{mM}$ CoA, $80 \mu \mathrm{M}$ Luciferin) and then with Renilla luciferase buffer $\left(1,1 \mathrm{M} \mathrm{NaCl}, 2.2 \mathrm{mM} \mathrm{Na}{ }_{2}\right.$ EDTA, $0.22 \mathrm{M}$ $\mathrm{K}_{2} \mathrm{HPO}_{4} \mathrm{pH} 5.1,0.5 \mathrm{mg} / \mathrm{ml}$ BSA, $1.5 \mathrm{mM} \mathrm{NaN3}, 1.5 \mu \mathrm{M}$ Coelenterazine). Relative light units (RLUs) were measured in a Luminometer Berthold Centro LB 960 plate reader. Values were normalized to Renilla activity and relativized to the control treatment.

\section{Murine organoids, media, culturing and treatment}


1047 For preparation of organoid media, HEK293T cells stably expressing mRspondin or mNoggin 1048 (kindly provided by Dr. Tiago De Oliveira), or mWnt3a cells were cultured in DMEM (Gibco) 1049 supplemented with GlutaMAX ${ }^{\mathrm{TM}}$ (Gibco), 10\% FBS (Merck), Penicillin-Streptomycin (10,000 $1050 \mathrm{U} / \mathrm{mL}$, Gibco) and Sodium Pyruvate (Gibco) in a humidified atmosphere at $37^{\circ} \mathrm{C}$ with $5 \% \mathrm{CO}_{2}$. 1051 For HEK293T mRpondin-I $300 \mu \mathrm{g} / \mathrm{mL}$ Zeocin (InvivoGen, \#ant-zn-05) and for HEK293T 1052 mNoggin $500 \mu \mathrm{g} / \mathrm{mL}$ G418 (Geneticin, InvivoGen, \#ant-gn-1) were added to the medium during 1053 cultivation. After HEK293 cell expansions, culturing media were replaced by conditioned 1054 medium (CM) containing Advanced DMEM/F-12 (Gibco) supplemented with GlutaMAX ${ }^{\mathrm{TM}}$ 1055 (Gibco), Penicillin-Streptomycin (10,000 U/mL, Gibco) and 10mM HEPES (Gibco). $50 \mathrm{~mL}$ of CM 1056 were added per $175 \mathrm{~cm}^{2}$ flask and HEK293 cells allowed to grow for one week. Each CM media 1057 were sterilly filtered and aliquoted. Since mRspondin-I and mNoggin proteins are each fused to 1058 an Fc-tag, the quality of each batch was tested by Dot-blot analysis. Organoid media was 1059 composed of 50\% CM Wnt3a, 20\% CM mNoggin, 10\% CM mRspondin-I, N2 and B27 (both

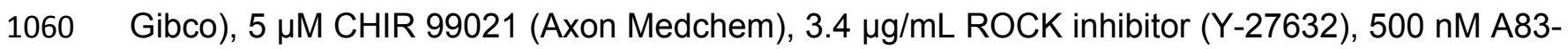
1061 01, $10 \mathrm{mM}$ Nicotinamide (Sigma-Aldrich), $80 \mu \mathrm{M} \mathrm{N}$-Acetyl-L-Cysteine (all Sigma-Aldrich), and $1062200 \mathrm{ng} / \mathrm{mL}$ rmEGF (ImmunoTools).

1063 For organoid preparation, tumor-harboring mice were sacrificed and the colons harvested. 1064 Tumors were dissected, washed and minced, and incubated with $2 \mathrm{mg} / \mathrm{mL}$ Collagenase type I 1065 solution (Gibco, dissolved in Advanced DMEM/F12) at $37^{\circ} \mathrm{C}$ for $30 \mathrm{~min}$, while pipetting up and 1066 down every $10 \mathrm{~min}$ to dissociate the tumors. Small tumor fragments were transferred into a new 1067 Falcon tube using a cell strainer (100 $\mu \mathrm{m}$ mesh size). Fragments were centrifuged and washed 1068 with Advanced DMEM/F12. After centrifugation, tumor fragments were resuspended in cold 1069 Matrigel (Corning) and plated as gel drops on culture plates. After Matrigel polymerization at $107037^{\circ} \mathrm{C}$, organoids were cultured in organoid media and cultivated in a humidified atmosphere at $107137^{\circ} \mathrm{C}$ with $5 \%$ CO2. Medium was exchanged every 2-3 days. Splitting of organoids was 1072 performed when organoids started to accumulate dead cells in the lumen (approx. once a 1073 week). To this end, organoids were recovered from Matrigel and disrupted manually by pipetting 1074 using $1 \mathrm{ml}$ blue tips. For enzymatic dissociation, organoids were incubated with $0.25 \%$ trypsin at $107537^{\circ} \mathrm{C}$ for $10 \mathrm{~min}$, washed with Advanced DMEM/F12, centrifuged and cultured as described 1076 above. Experiments with murine colonic organoids were done between passage 3 and 8. 1077 p53LOH was induced with $1 \mu \mathrm{M} 4 \mathrm{OHT}$ (Sigma) for 24 - 48 hrs (as indicated in the figure 1078 legends) in CHIR 99021-free and Rock-free organoid media. 


\section{Immunofluorescence staining of organoids}

1081 Organoids were fixed within Matrigel domes with 2\% / 0.1\% Paraformaldehyde/Glutaraldehyde 1082 /PBS for 30 min. After intensively washing steps with PBS, gel domes with fixed organoids were 1083 removed from the plate and transferred into a tube. Sucrose infiltration was started with $20 \%$ 1084 sucrose / PBS, followed by $40 \%$ sucrose / PBS, each incubated over night or longer at $4^{\circ} \mathrm{C}$ until 1085 the domes settled down. After sucrose infiltration, organoids were embedded in TissueTEK 1086 (Tissue-Tek ${ }^{\circledR}$ O.C. $T^{\mathrm{TM}}$ Compound) and $10 \mu \mathrm{M}$ cryo-sections were cut. Sections were air-dried 1087 for $30 \mathrm{~min}$ at RT, pre-wetted with PBS and quenched with $10 \mathrm{mM} \mathrm{NaBH} 4$ / PBS twice for 5 1088 minutes at room temperature each time. After washing steps, samples were permeabilised with $10890.1 \%$ TritonX-100 / PBS for 10 min at RT and blocked with 10\% FBS / 1\% BSA / PBS for 1 hour. 1090 For staining, samples were co-incubated with the p53 antibody FL393 (Santa Cruz) and E1091 Cadherin (BD Biosciences) overnight at $4^{\circ} \mathrm{C}$. Primary antibodies were detected by 1092 AlexaFluor488- and AlexaFluor647- conjugated secondary antibodies (Molecular Probes). 1093 Organoids were DAPI counterstained and mounted in Fluoromount media (DAKO). Images were taken using a standard fluorescence microscope (Carl Zeiss AG) with the ZEN imaging program from Zeiss. Figures were further prepared using Adobe Photoshop software.

\section{Analysis of human patient TCGA data}

1098 We used TCGA (The Cancer Genome Atlas) colorectal cancer (COARDREAD) and breast 1099 cancer (BRCA) databases in this analysis. Human genomic data including RNA expression, 1100 DNA copy number alteration, gene mutation, and clinical information was downloaded from 1101 cBioPortal for cancer genomics (http://www.cbioportal.org). Study names: Colorectal 1102 adenocarcinoma (TCGA, PanCancer Atlas, 594 total samples) and Breast Invasive Carcinoma 1103 (TCGA, PanCancer Atlas, 1084 total samples). TP53 wild type (WTp53) group are those 1104 samples without TP53 mutations. TP53 missense mutant group was samples with TP53 1105 missense mutations (MS), and TP53 LOF group was determined by samples with all TP53 1106 mutations (MS, missense; FS, frameshift; NS, nonsense). To identify tumors harboring p53LOH, 1107 we selected samples that had both a mutated TP53 gene and a shallow deletion in DNA copy 1108 number. The list of HSF1 target genes was chosen from Mendillo et al. ${ }^{7}$ We compared the 1109 expression values (by RNAseq) of HSF1 target genes from mutant p53/p53LOH tumors with 1110 samples that harbored wildtype TP53 $\left(\right.$ TP53 $\left.^{+/+}\right)$. Further we applied survival analysis to check 1111 patients with a missense TP53 mutation (MS p53) and a p53LOH compared to a WTp53 patient 1112 group. R language (The R Project for Statistical Computing, https://www.r-project.org) was used 1113 in the analysis. R package "gplots" was used to generate heatmaps. R package "survival" were 
used for survival analysis, including calculating log-rank p-values and generating Kaplan-Meier

1115 curves.

\section{QUANTIFICATION AND STATISTICAL ANALYSIS}

1119 Statistics of each experiment such as number of animals, number of tumors, biological 1120 replicates, technical replicates, precision measures (mean and $\pm S E M$ ) and the statistical tests 1121 used for significance are provided in the figures and figure legends.

1122 Unpaired Student's t test was used to calculate the $\mathrm{p}$ values for comparisons of tumor numbers 1123 and sizes and mRNA expression levels.

1124 Densitometric measurements for quantification of immunoblot bands were done with the gel 1125 analysis software Image Lab $^{\mathrm{TM}}$ (BioRad) and normalized to loading controls.

1126 The following designations for levels of significance were used within this manuscript: $p^{*}=0.05$;

$1127 p^{* *}=0.01 ; p^{* * *}=0.001 ; n s$, not significant.

1.Hanel, W. et al. Two hot spot mutant p53 mouse models display differential gain of function in tumorigenesis. Cell Death Differ 20, 898-909 (2013). for treatment. Nature 523, 352-356 (2015). 3.Schulz-Heddergott, R. et al. Therapeutic Ablation of Gain-of-Function Mutant p53 in Colorectal Cancer Inhibits Stat3-Mediated Tumor Growth and Invasion. Cancer Cell 34, 298-314 e297 (2018).

1136

1137 4.Jonkers, J. et al. Synergistic tumor suppressor activity of BRCA2 and p53 in a conditional mouse model for breast cancer. Nat Genet 29, 418-425 (2001).

1138 5.Jacks, T. et al. Tumor spectrum analysis in p53-mutant mice. Curr Biol 4, 1-7 (1994). 6.Becker, C., Fantini, M.C. \& Neurath, M.F. High resolution colonoscopy in live mice. Nature protocols 1, 2900-2904 (2006). 
Table 1, related to online Methods:: Reagents and Resources

\begin{tabular}{|c|c|c|c|}
\hline REAGENT or RESOURCE & SOURCE & \multicolumn{2}{|c|}{ IDENTIFIER } \\
\hline \multicolumn{4}{|l|}{ Antibodies } \\
\hline Rabbit polyclonal anti-p53 (FL-393) & Santa Cruz & \multicolumn{2}{|c|}{ Cat\# sc-6243; RRID:AB_653753 } \\
\hline Goat polyclonal anti-aSMA & Abcam & \multicolumn{2}{|c|}{ Cat\# ab21027, RRID:AB_1951138 } \\
\hline Mouse monoclonal anti-E-Cadherin & BD Biosciences & \multicolumn{2}{|c|}{ Cat\# 610181; RRID:AB_397580 } \\
\hline Mouse monoclonal anti-p53 (DO-1) & Santa Cruz & \multicolumn{2}{|c|}{ Cat\# sc-126; RRID:AB_628082 } \\
\hline Rabbit monoclonal phospho-Ser326-HSF1 & Abcam & \multicolumn{2}{|c|}{ Cat\# ab76076; RRID:AB_1310328 } \\
\hline Rabbit polyclonal anti-HSF1 (H-311) & Santa Cruz & \multicolumn{2}{|c|}{ Cat\# sc-9144; RRID:AB_2120276 } \\
\hline Rabbit monoclonal anti-HSP27 (E1J4D) & Cell Signaling & \multicolumn{2}{|c|}{ Cat\# 50353; RRID:AB_2799374 } \\
\hline $\begin{array}{l}\text { Rabbit polyclonal anti-Heat Shock Protein } \\
\text { 90alpha }\end{array}$ & Millipore & \multicolumn{2}{|c|}{$\begin{array}{l}\text { Cat\# 07-2174; } \\
\text { RRID:AB_10807022 }\end{array}$} \\
\hline Rabbit polyclonal anti-AKT & Cell Signaling & \multicolumn{2}{|c|}{ Cat\# 9272; RRID:AB_329827 } \\
\hline Mouse monoclonal anti-beta-actin & Abcam & \multicolumn{2}{|c|}{ Cat\# ab6276; RRID:AB_2223210 } \\
\hline Rabbit polyclonal anti-c-Raf & Cell Signaling & \multicolumn{2}{|c|}{ Cat\# 9422; RRID:AB_390808 } \\
\hline Rabbit monoclonal anti-p21 Waf1/Cip1 (12D1) & Cell Signaling & \multicolumn{2}{|c|}{ Cat\# 2947; RRID:AB_823586 } \\
\hline $\begin{array}{l}\text { Rabbit monoclonal anti-phospho-Rb } \\
\text { (Ser807/811) (D20B12) XP }\end{array}$ & Cell Signaling & \multicolumn{2}{|c|}{ Cat\# 8516; RRID: AB_11178658 } \\
\hline $\begin{array}{l}\text { Rabbit polyclonal anti-phospho-Ser235/236- } \\
\text { S6 ribosomal protein }\end{array}$ & Cell Signaling & \multicolumn{2}{|c|}{ Cat\# 2211; RRID:AB_331679 } \\
\hline Rabbit monoclonal anti-MLK3 [EP1460Y] & Abcam & \multicolumn{2}{|c|}{ Cat\# ab51068; RRID:AB_881140 } \\
\hline $\begin{array}{l}\text { Rabbit polyclonal phospho-p-MEK-1/2 (Ser } \\
218 / \text { Ser 222) }\end{array}$ & Santa Cruz & \multicolumn{2}{|c|}{ Cat\# sc-7995; RRID:AB_2234805 } \\
\hline Mouse monoclonal anti-GAPDH & Abcam & \multicolumn{2}{|c|}{ Cat\# ab8245; RRID:AB_2107448 } \\
\hline Mouse monoclonal anti-Cdc2 p34 & Santa Cruz & \multicolumn{2}{|c|}{ Cat\# sc-54; RRID:AB_627224 } \\
\hline Rabbit monoclonal anti-CDK2 & Abcam & \multicolumn{2}{|c|}{ Cat\# ab32147; RRID:AB_726775 } \\
\hline Rabbit polyclonal anti-PLK4 & $\begin{array}{l}\text { Protein } \\
\text { Technologies }\end{array}$ & \multicolumn{2}{|c|}{$\begin{array}{l}\text { Cat\# 12952-1-AP; } \\
\text { RRID:AB_2284150 }\end{array}$} \\
\hline Alexa Fluor®488 Goat anti-rabbit IgG $(\mathrm{H}+\mathrm{L})$ & ThermoFisher & \multicolumn{2}{|c|}{ Cat\# A-11034; RRID:AB_2576217 } \\
\hline $\begin{array}{l}\text { Alexa Fluor®488 Donkey anti-mouse IgG } \\
(\mathrm{H}+\mathrm{L})\end{array}$ & ThermoFisher & \multicolumn{2}{|c|}{ Cat\# A-21202; RRID:AB_141607 } \\
\hline Alexa Fluor®546 Donkey anti-rabbit lgG $(\mathrm{H}+\mathrm{L})$ & ThermoFisher & \multicolumn{2}{|c|}{ Cat\# A-10040; RRID:AB_2534016 } \\
\hline ImmPRESS ${ }^{\mathrm{TM}}$ Peroxidase polymer reagent & VectorLabs & \multicolumn{2}{|c|}{ Cat\# MP-7401, RRID:AB_2336529 } \\
\hline \multicolumn{4}{|l|}{ Bacterial and Virus Strains } \\
\hline Bacteria: ElectroMAX DH10B cells & nvitrogen/Thermo F & er Sci. & Cat\# 18290-015 \\
\hline Chemicals, Peptides, and Recombinant P & teins & & \\
\hline AOM (Azoxymethane) & Sigma Aldrich & & Cat\# A5486 \\
\hline DSS (Dextran sodium sulfate) & MP Biomedicals & & Cat\# 160110 \\
\hline TAM (Tamoxifen) & Sigma Aldrich & & Cat\# T5648 \\
\hline (Z)-4-Hydroxytamoxifen (4-OHT) & Sigma Aldrich & & Cat\# H7904 \\
\hline Lipofectamine2000 & Invitrogen & & Cat\# 11668-019 \\
\hline Trizol & Invitrogen & & Cat\# 15596026 \\
\hline Phusion ${ }^{\circledR}$ High-Fidelity DNA Polymerase & Thermo Fisher Sc & & Cat\# F530 \\
\hline PD 0332991 isethionate (Palbociclib) & Sigma Aldrich & & Cat\# PZ0199 \\
\hline RO-3306 & Sigma Aldrich & & Cat\# SML0569 \\
\hline Roscovitine & Cell Signaling & & Cat\# 9885 \\
\hline RG-7112 & SelleckChem & & Cat\# S7030 \\
\hline
\end{tabular}




\begin{tabular}{|c|c|c|c|c|}
\hline Idasanutlin (RG-7388) & & \multicolumn{2}{|l|}{ SelleckChem } & Cat\# S7205 \\
\hline Nutlin-3a (MDM2 inhibitor) & & \multicolumn{2}{|l|}{ BOC Sciences } & Cat\# 675576-98-4 \\
\hline Passive Lysis Buffer, $5 \mathrm{X}$ & & \multicolumn{2}{|l|}{ Promega } & Cat\# E194A \\
\hline \multicolumn{5}{|l|}{ Experimental Models: Cell Lines } \\
\hline HCT116 & \multicolumn{3}{|c|}{ ATCC } & Cat\# ATCC $®$ CCL-247 TM \\
\hline HCT116-ORF & \multicolumn{3}{|c|}{ this work } & $\mathrm{N} / \mathrm{A}$ \\
\hline HCT116-HSF1c1 & \multicolumn{3}{|c|}{ this work } & $\mathrm{N} / \mathrm{A}$ \\
\hline HCT116-HSF1c2 & \multicolumn{3}{|c|}{ this work } & $\mathrm{N} / \mathrm{A}$ \\
\hline RKO & \multicolumn{3}{|c|}{ ATCC } & Cat\# ATCC $®$ CRL-2577 ${ }^{\text {TM }}$ \\
\hline LS513 & \multicolumn{3}{|c|}{ ATCC } & Cat\# ATCC $®$ CRL-2134 ${ }^{\mathrm{TM}}$ \\
\hline HCT116 p53-/- & \multicolumn{3}{|c|}{ Bunz et al., 1998.} & B. Vogelstein, Baltimore \\
\hline HCT116 p53+/+ & \multicolumn{3}{|c|}{ Bunz et al., 1998.} & B. Vogelstein, Baltimore \\
\hline LS174T & \multicolumn{3}{|c|}{ DSMZ } & Cat\# ACC 759 \\
\hline SW480 & \multicolumn{3}{|c|}{ DSMZ } & Cat\# ACC 313 \\
\hline MCF-7 & \multicolumn{3}{|c|}{ DSMZ } & Cat\# ACC 115 \\
\hline MDA-MB-231 & \multicolumn{3}{|c|}{ DSMZ } & Cat\# ACC 732 \\
\hline $\begin{array}{l}\text { HEK } 293 \text { Cell Line human (for viral } \\
\text { transfection) }\end{array}$ & \multicolumn{3}{|c|}{ DSMZ } & Cat\# 85120602 \\
\hline \multicolumn{5}{|l|}{ Experimental Models: Organisms/Strains } \\
\hline Mouse: $p 53^{\text {LoxP }}\left(p 53^{f f}\right)$ & \multicolumn{3}{|c|}{ Jonkers J et al., 2001} & Jax strain\# 008462 \\
\hline $\begin{array}{l}\text { Mouse: p53null (-/-) (B6.129S2- } \\
\text { Trp53<tm1Tyj>/J) }\end{array}$ & \multicolumn{3}{|c|}{$\begin{array}{l}\text { Jacks T et al., } 1994 \text { or The } \\
\text { Jackson Laboratory }\end{array}$} & Jax strain\# 002101 \\
\hline Mouse: $\mathrm{p} 53^{\mathrm{R} 248 \mathrm{Q}}$ & \multicolumn{3}{|c|}{ Hanel et al., 2013} & N/A \\
\hline Mouse: $p 53^{\text {floxR248Q }}\left(p 53^{f l o x Q}\right)$ & \multicolumn{3}{|c|}{ Alexandrova et al., 2015} & $\mathrm{~N} / \mathrm{A}$ \\
\hline Mouse: villin:CreER ${ }^{\frac{1}{2} 2}$ & \multicolumn{3}{|c|}{$\mathrm{N} / \mathrm{A}$} & Jax strain\# 020282 \\
\hline Mouse: C57BL/6NJ & \multicolumn{3}{|c|}{$\mathrm{N} / \mathrm{A}$} & Jax strain\# 005304 \\
\hline \multicolumn{5}{|l|}{ Oligonucleotides } \\
\hline Primers for QPCR and genotyping, see below & & this paper & & Table S1. \\
\hline siRNA MLK3 Silencer® Select & & Ambion & & Pool of IDs: $8814+8815+8816$ \\
\hline siRNA CDC2 Silencer® Select & & Ambion & & ID: 464 \\
\hline siRNA CDC2 Silencer® Select & & Ambion & & ID: 465 \\
\hline siRNA CDK2 Silencer® Select & & Ambion & & ID: 205 \\
\hline siRNA CDK2 Silencer® Select & & Ambion & & ID: 206 \\
\hline siRNA PLK4 Silencer® Select & & Ambion & & ID: 21083 \\
\hline siRNA PLK4 Silencer@ Select & & Ambion & & ID: 21084 \\
\hline siRNA TP53 Silencer® Select & & Ambion & & ID: s605 \\
\hline siRNA TP53 Silencer $囚$ Select & & Ambion & & ID: s607 \\
\hline siRNA CDKN1A Silencer® Select & & Ambion & & ID: 415 \\
\hline siRNA CDKN1A Silencer® Select & & Ambion & & ID: 417 \\
\hline $\begin{array}{l}\text { siRNA Negative Control No. } 2 \text { (src2) Silencer } \\
\text { Select siRNA }\end{array}$ & er® & Ambion & & Cat\# 4390847 \\
\hline Recombinant DNA & & & & \\
\hline pSUPER control vector for shRNA & $\overline{O l i g o}$ & oEngine & Cat\# V & VEC-PBS-0002 \\
\hline pSUPER-p53 for shp53 & Oligo & oEngine & Cat\# V & VEC-P53-0001 \\
\hline pMD2.G & Addg & gene & Plasm & id \#12259 \\
\hline pCMV-R8.91 & $\begin{array}{l}\text { Plasn } \\
\text { Biele }\end{array}$ & $\begin{array}{l}\text { midFactory } \\
\text { efeld }\end{array}$ & Krame & er et al., 2017. PMID: 27834954 \\
\hline Precision LentiORF positive control & Dhar & rmacon & & \\
\hline
\end{tabular}




\section{0}

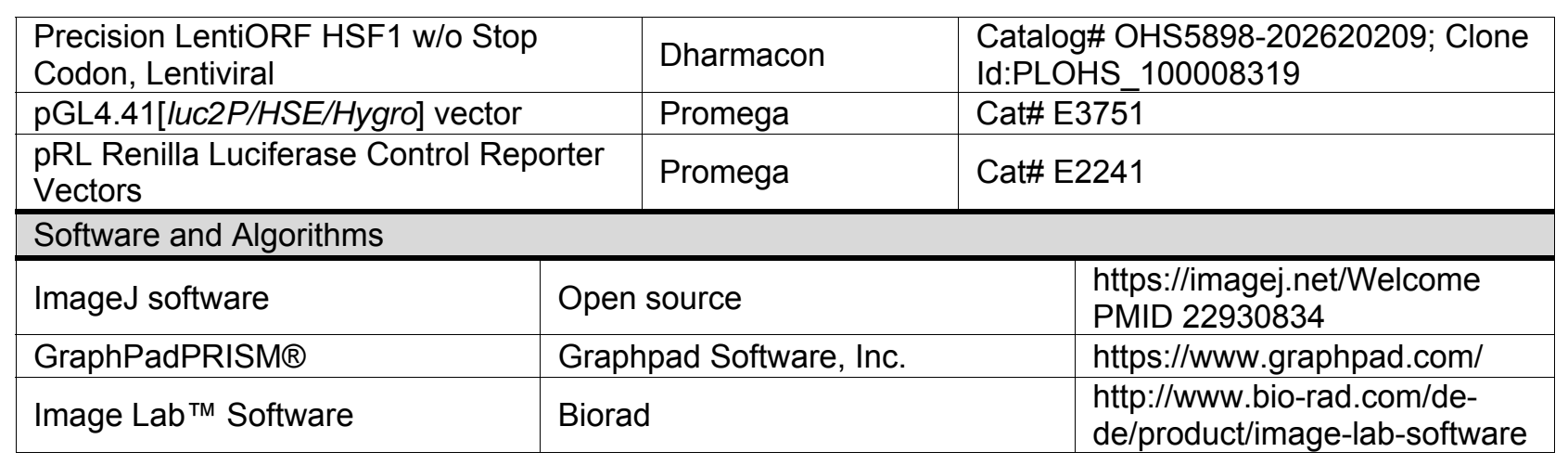

1151

1152

1153

1154

1155

1156

1157

1158

1159

1160

1161

1162

1163

1164

1165

1166

1167

1168

1169 
1170 Table S2, related to online Methods: Primers for qPCR and genotyping.

\begin{tabular}{|c|c|c|c|c|c|}
\hline Gene & Origin & \multicolumn{2}{|c|}{ Forward } & \multicolumn{2}{|c|}{ Reverse } \\
\hline \multicolumn{6}{|l|}{ qPCR } \\
\hline HSP90AA1 & Human & \multicolumn{2}{|c|}{ 5'-GCCCAGAGTGCTGAATACCC } & \multicolumn{2}{|c|}{ 5'-GTGGAAGGGCTGTTTCCAGA } \\
\hline HSPA1A & Human & \multicolumn{2}{|c|}{ 5'-TCAAGGGCAAGATCAGCGAG } & \multicolumn{2}{|c|}{ 5'-TGATGGGGTTACACACCTGC } \\
\hline HSPH1 & Human & \multicolumn{2}{|c|}{ 5'-ACTGCTTGTTCAAGAGGGCTGTGA } & \multicolumn{2}{|c|}{ 5'-AACATCCACACCCACACACATGCT } \\
\hline HSPB1 & Human & \multicolumn{2}{|c|}{ 5'-GGAGTGGTCGCAGTGGTTAG } & \multicolumn{2}{|c|}{ 5'-ATGTAGCCATGCTCGTCCTG } \\
\hline CDC6 & Human & \multicolumn{2}{|c|}{ 5'-TAAAAGCCCTGCCTCTCAGC } & \multicolumn{2}{|c|}{ 5'-TGAGTGAGGGGGACCATTCT } \\
\hline ITGB3BP & Human & \multicolumn{2}{|c|}{ 5'-TCCCGAATCTCAGAATGCCTG } & \multicolumn{2}{|c|}{ 5'-TGACAAGTTCCAGTTGTTGGAG } \\
\hline RBBP5 & Human & \multicolumn{2}{|c|}{ 5'-AACTCAGCCAGCCCTTGAC } & \multicolumn{2}{|c|}{ 5'-GGCCACATGATGGCAAAGTG } \\
\hline BST2 & Human & \multicolumn{2}{|c|}{ 5'-AGGAGCTTGAGGGAGAGATCA } & \multicolumn{2}{|c|}{ 5'- AGGACGGACCTTCCAAGATG } \\
\hline$R P L P O$ (36B4) & Human & \multicolumn{2}{|c|}{ 5'-GATTGGCTACCCAACTGTTG } & \multicolumn{2}{|c|}{ 5'-CAGGGGCAGCAGCCACAAA } \\
\hline TP53 & Human & \multicolumn{2}{|c|}{ 5'-AAGTCTAGAGCCACCGTCCA } & \multicolumn{2}{|c|}{ 5'-CAGTCTGGCTGCCAATCCA } \\
\hline FBNL1 & Human & \multicolumn{2}{|c|}{ 5'-CCGCAACTGCCAAGACATTGAT } & \multicolumn{2}{|c|}{ 5'-GACCGTGTCTGTCTTCTCCTG } \\
\hline$C D K N 1 A$ & Human & \multicolumn{2}{|c|}{ 5'-TAGGCGGTTGAATGAGAGG } & 5 -AA & GTGGGGAGGAGGAAGTAG \\
\hline$C D K 1$ & Human & 5'-TTT & CAGAGCTTTGGGCACT & 5 -CC & ATTTTGCCAGAAATTCGT \\
\hline$C D K 2$ & Human & 5'-GGA & TGCCTCTGCTCTCACTG & 5 -AC & AGGGTCACCACCTCATGG \\
\hline$C D C 25 c$ & Human & 5'-GTA & TCTGGGAGGACACATCCAGGG & 5 -CA & AGTTGGTAGCCTGTTGGTTTG \\
\hline PLK4 & Human & 5'-CAA & GCGGCGGGAGATTTTCA & 5 -CA & GCTCTGTAGACACCAGCAA \\
\hline$M L K 3$ & Human & 5 '-CAC & ACCCCCAGCACTCAAT & 5 -CC & TCTTGAGCGAGAAGCAGA \\
\hline Trp53 (Ex1-Ex3) & Mouse & 5'-GTC & CTCACCCTGGCTAAAGT & 5 5'-CA & GTGAGGTGATGGCAGGAT \\
\hline Ccnd1 & Mouse & 5 '-GGA & GCTGCTGCAAATGGAAC & 5 -CA & GTCCGGGTCACACTTGA \\
\hline Ccnb1 & Mouse & 5'-CAC & GGTCGTGAAGTGACTGG & 5'-GC & CACACAACTGTTCTGCAT \\
\hline Mdm2 & Mouse & 5'-TAG & CAG CCA AGA AAG CGT GA & 5'-AT & G AGG TGT CCA GTC TTG CC \\
\hline Pcna & Mouse & 5'-AGT & GGAGAGCTTGGCAATGG & 5 -TC & AGGTACCTCAGAGCAAACG \\
\hline Cdkn1a (p21) & Mouse & 5'-GTC & GCCTTGTCGCTGTCTT & $5 '-G$ & SGTTGGAGTGATAGAAATCTG \\
\hline Gadd45a & Mouse & 5'-GGC & GTGTACGAGGCTGCCAA & 5 -TC & TCGTTCTCGCAGCAGAACG \\
\hline$B b c 3$ & Mouse & 5'-TTC & TCCGGAGTGTTCATGCC & 5 -AT & ACAGCGGAGGGCATCAGG \\
\hline Sfn & Mouse & 5 -GCC & CGGTCAGCCTACCAGGA & 5 -CC & GCTGTCCACAGCGTCAGG \\
\hline $\mathrm{HspH1}$ & Mouse & 5'-AGA & CCA TCG CCA ACG AGT TC & 5 5'AC & A TGA CCT TTA TTC CCA CGC \\
\hline HspE & Mouse & 5'-GGA & GTG CTG CCG AAA CTG TA & 5 - C & A ACT TTC ACA CTG ACA GGC \\
\hline Hsp90AA1 & Mouse & 5'-CGT & CTC GTG CGT GTT CAT TC & 5 -CC & A GAG CGT CCG ATG AAT TG \\
\hline Itgb3bp & Mouse & 5'-GTA & TAC AGG CTT TGG AGG GCA & 5 -TC & A CAG TTG TCA GAC TTG AAG GT \\
\hline pre-miR34a & Mouse & 5'-GG1 & AGGGTCCACTACACATCTTTC & 5 -CT & AGGGCAGTATACTTGCTGATTG \\
\hline Snai1 & Mouse & 5'-CTT & STGTCTGCACGACCTG & 5 -GC & TTGGAGCGGTCAGCAAA \\
\hline Vim & Mouse & 5'-GGA & TCAGCTCACCAACGACA & 5 -AA & GGTCAAGACGTGCCAGAG \\
\hline Hprt1 & Mouse & 5'-GCT & TCC TCC TCA GAC CGC TT & 5 -CC & A GCA GGT CAG CAA AGA ACT \\
\hline RplpO (36B4) & Mouse & 5'-GCA & GATCGGGTACCCAACTGTT & 5 -CA & GCAGCCGCAAATGCAGATG \\
\hline genotyping & & & & & \\
\hline $\operatorname{Trp5} 3^{\mathrm{R} 248 \mathrm{Q}}=\mathrm{WT}$ & & Mouse & 5'-GGAAGTCCTTTGCCCTGAA & & 5'-CACTGAAAAAGACCTGGCAACC \\
\hline$T_{P} 53^{R 248 Q}=$ huma & $\mathrm{ed} Q$ & Hu/Mus & 5'-AAGGGTGCAGTTATGCCTC/ & man) & $\sim$ \\
\hline
\end{tabular}


bioRxiv preprint doi: https://doi.org/10.1101/2020.04.23.057034; this version posted April 24, 2020. The copyright holder for this preprint (which was not certified by peer review) is the author/funder. All rights reserved. No reuse allowed without permission.

\begin{tabular}{|l|l|l|l|}
\hline Trp53 $(\mathrm{X6-X7})=$ WT & Mouse & 5'-AGCGTGGTGGTACCTTATGAGC & 5'-GGATGGTGGTATACTCAGAGCC \\
\hline Trp53 $($ neo-X7) $=$ Del & Mouse & 5'-GCTATCAGGACATAGCGTTGGC & $\sim$ \\
\hline villinCreER $^{\text {I2 }}=$ transgene & Mouse & 5'-CAA GCC TGG CTC GAC GGC C & 5'- CGC GAA CAT CTT CAG GTT CT \\
\hline Trp53 $^{\text {tlox }}=$ WT and floxed & Mouse & 5'- GGT TAA ACC CAG CTT GAC CA & 5- GGA GGC AGA GAC AGT TGG AG \\
\hline
\end{tabular}

1171

1172

1173 
A
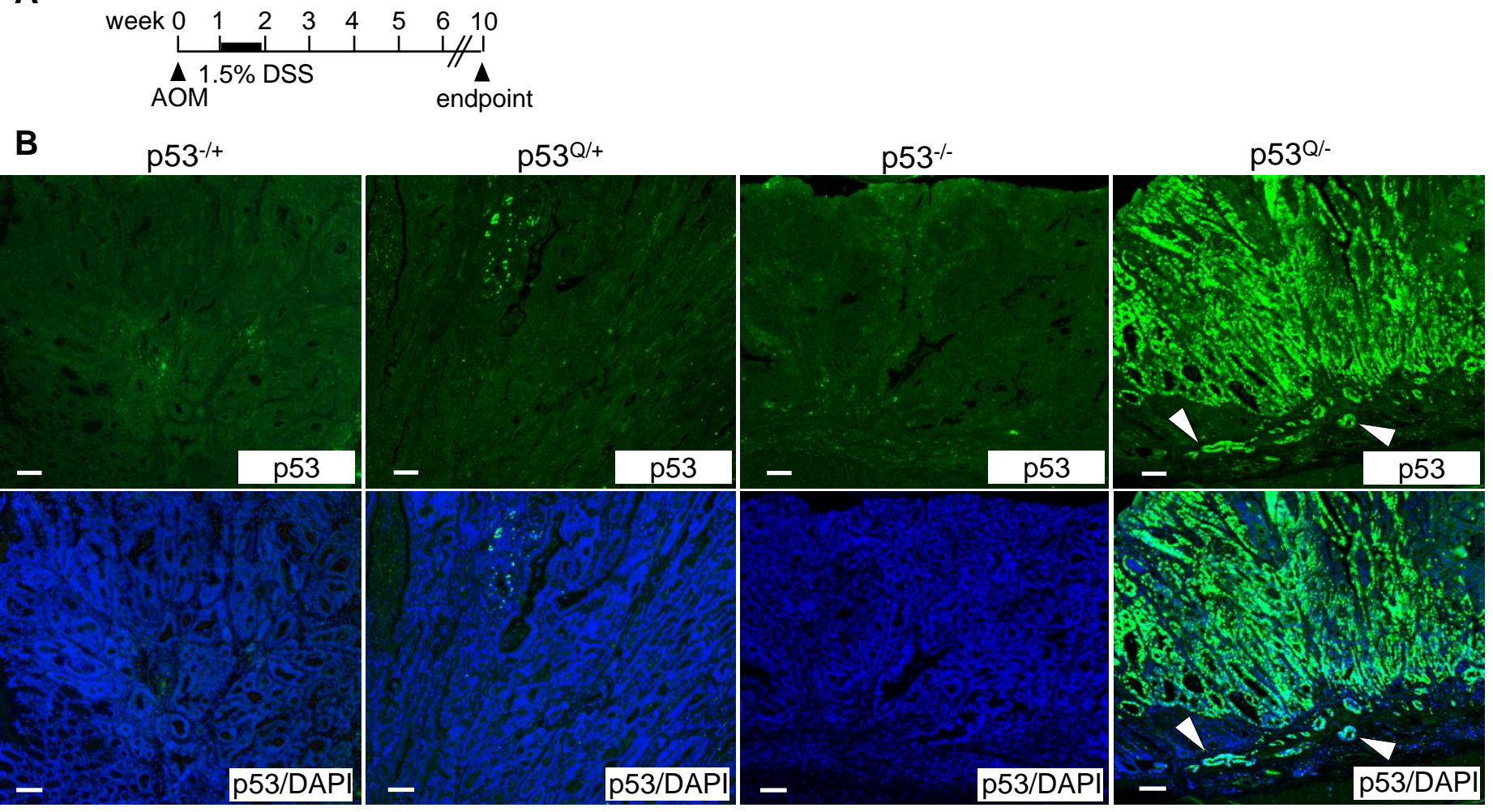

C

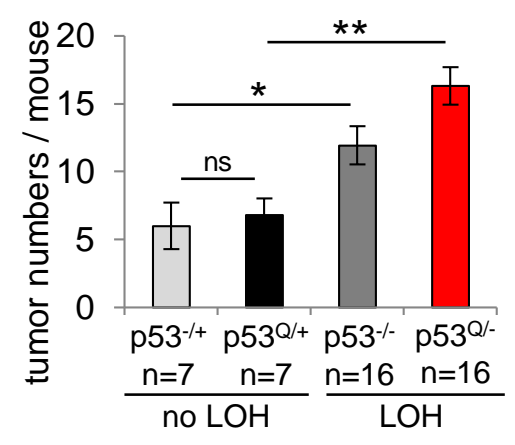

E

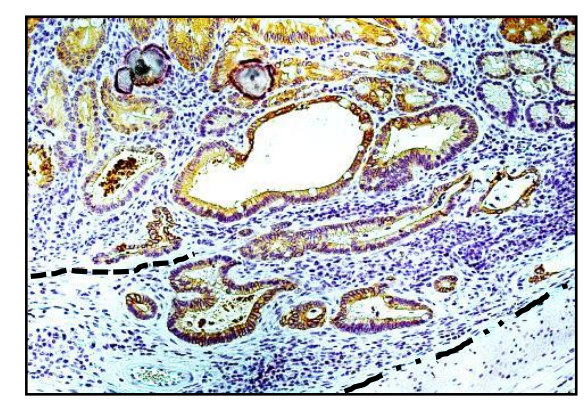

...-.-.-Muscularis mucosae

-..- Muscularis propria
D
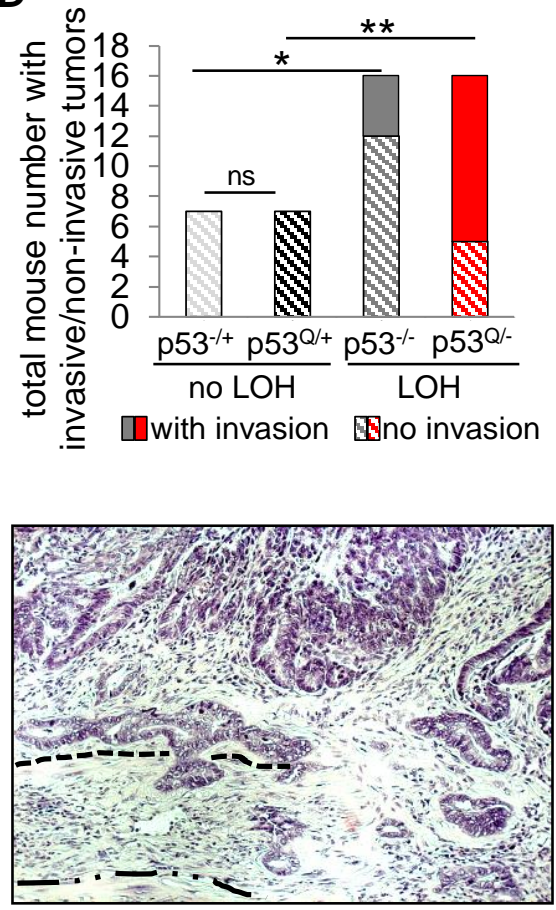

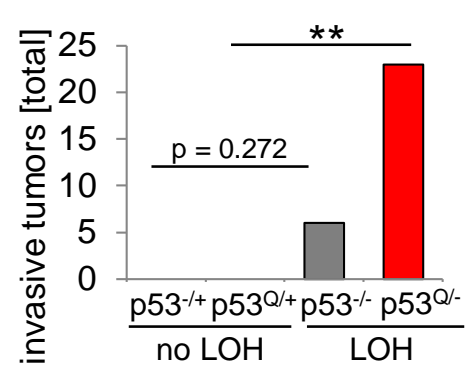

Supp Fig. 1 
A

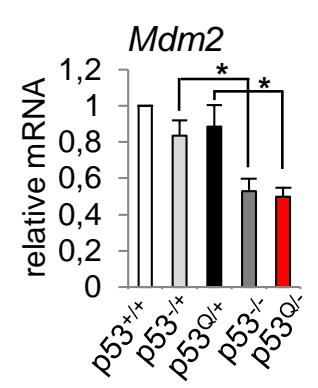

B

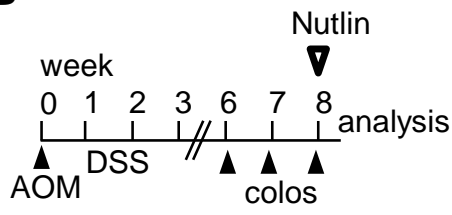

C

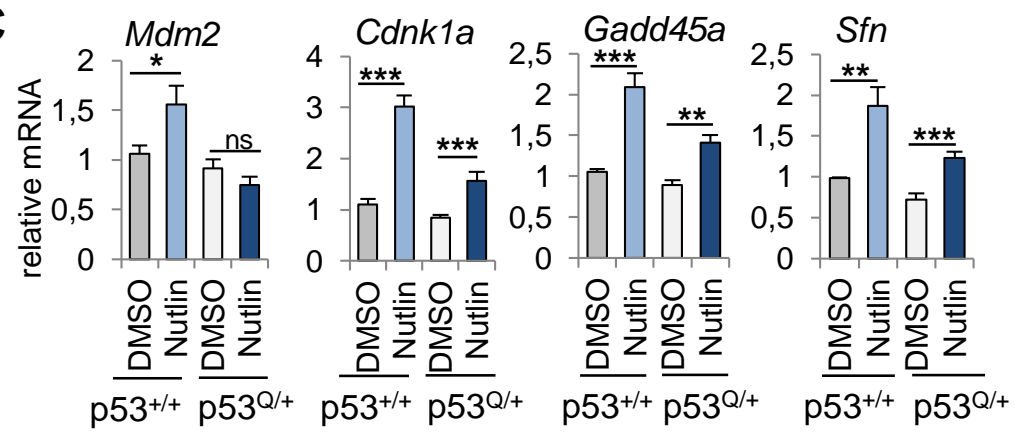

D

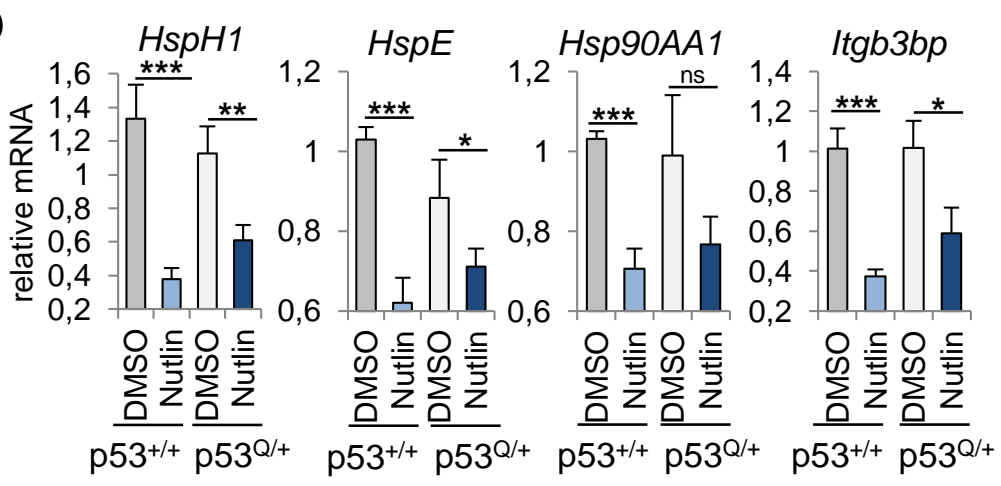

Figure 2 
A

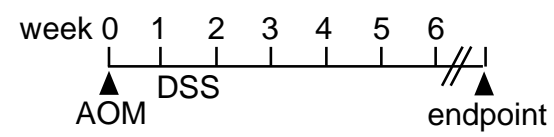

p53-deficient mice: $1.5 \%$ DSS with endpoint 10 wks p53-proficient mice: $1.8 \%$ DSS with endpoint 12 wks
B
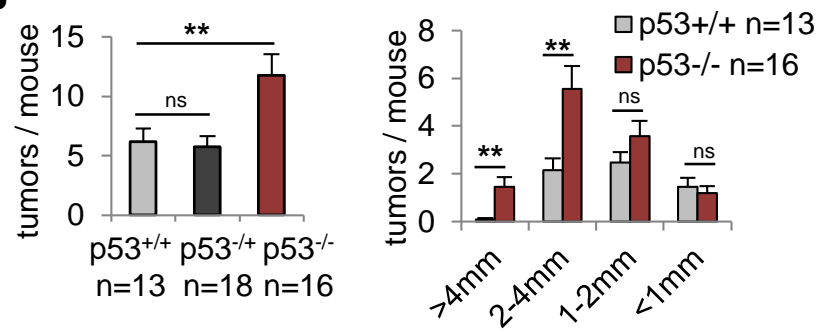

D

p53 $+/+$

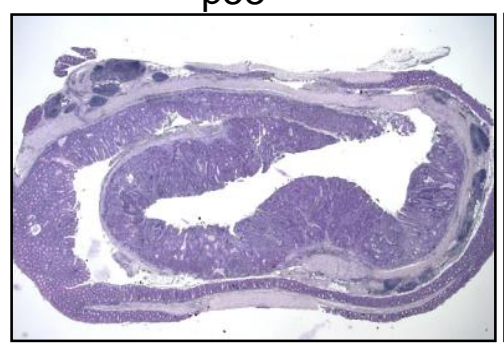

p53 ${ }^{--}$

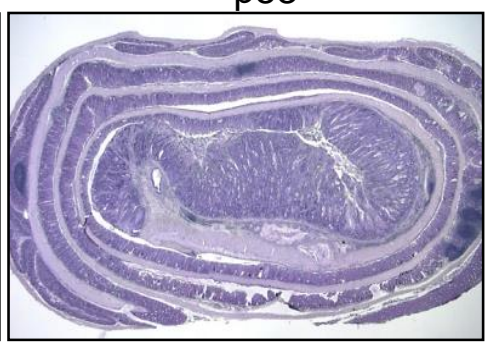

E

E

Pcna Conb1 $1_{* *} \quad$ Cond 1

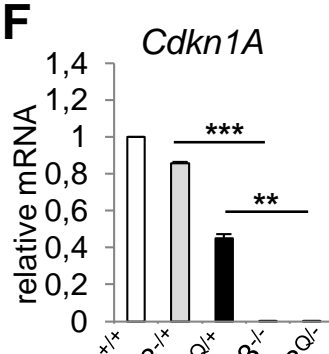

$B b c 3$

Gadd45a

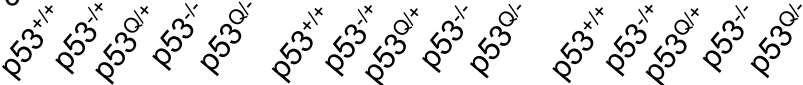
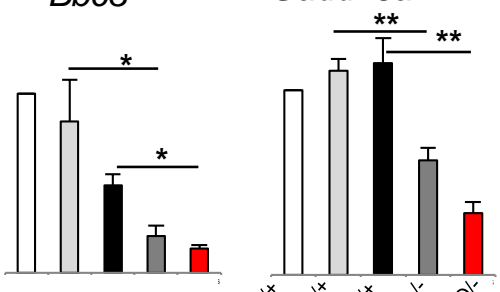

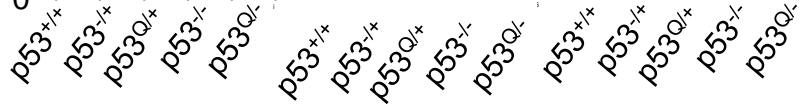

G

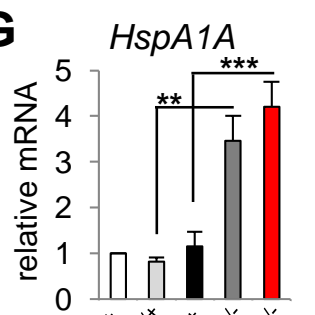

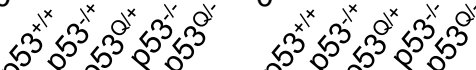

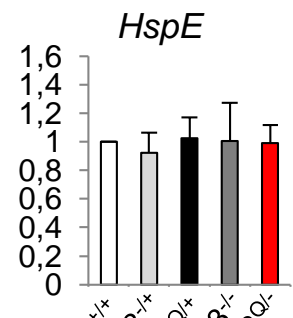

Hsp90AA1 Itgb3bp

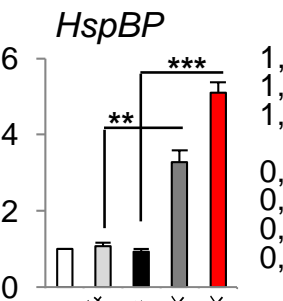

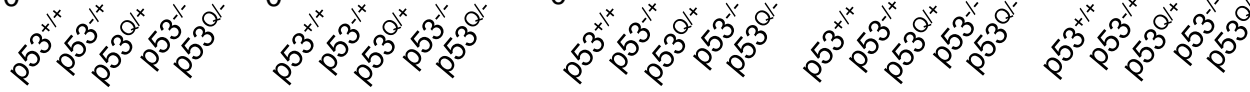
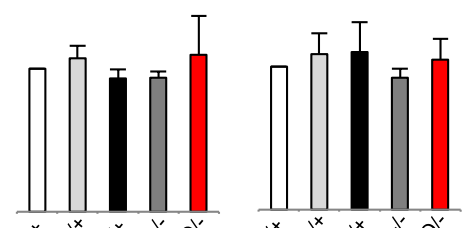

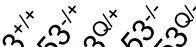

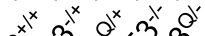



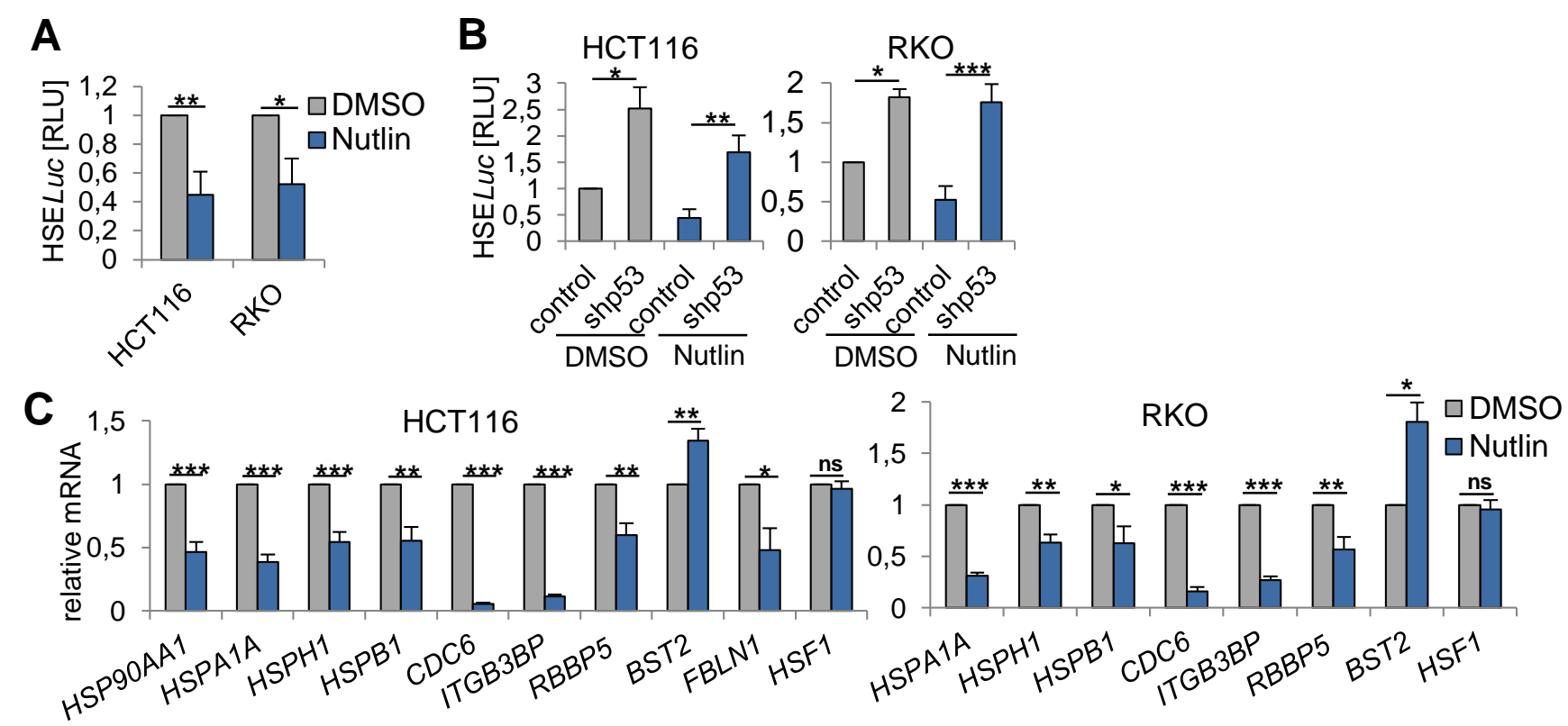

\section{D}

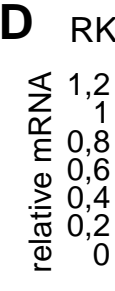

E

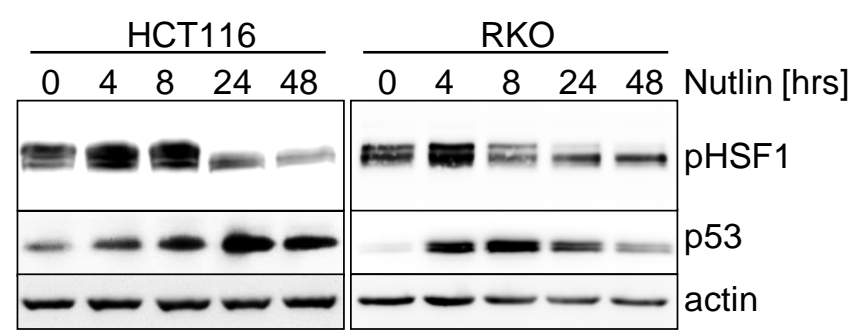

H

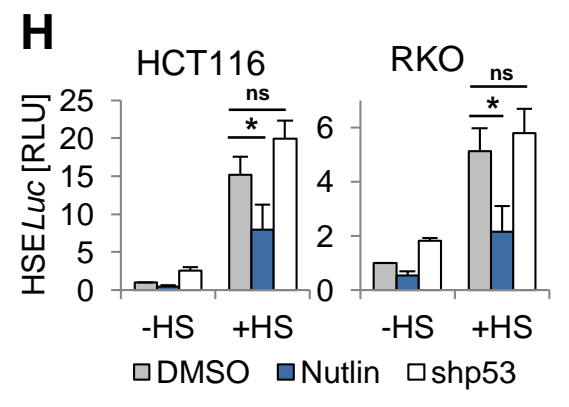

low exp

pHSF1

high exp

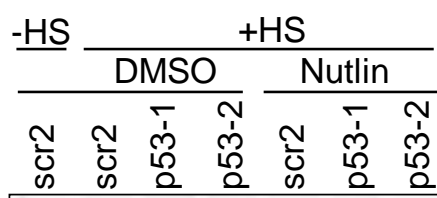

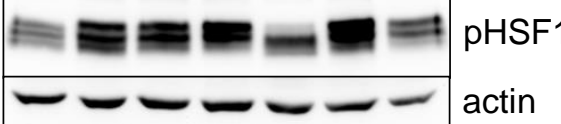

HCT116

Figure 3 
A HCT116

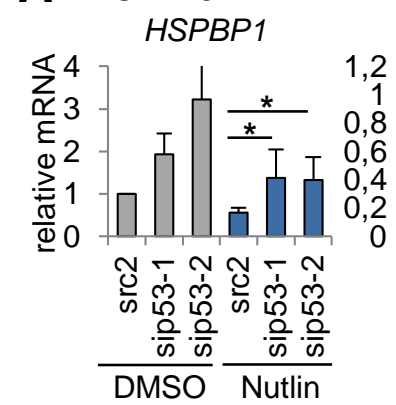

C

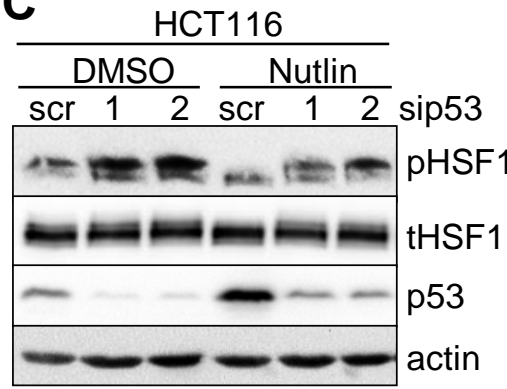

HSPA1A

CDC6

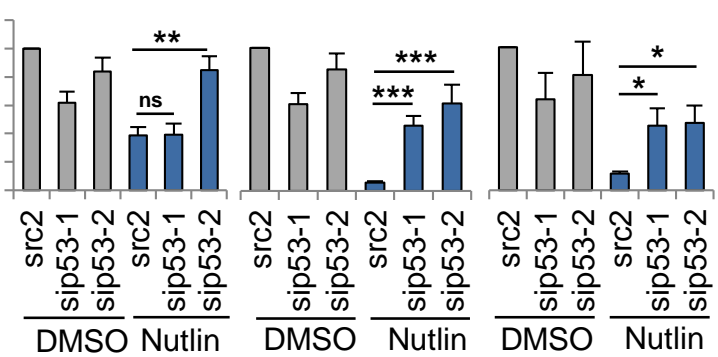

D

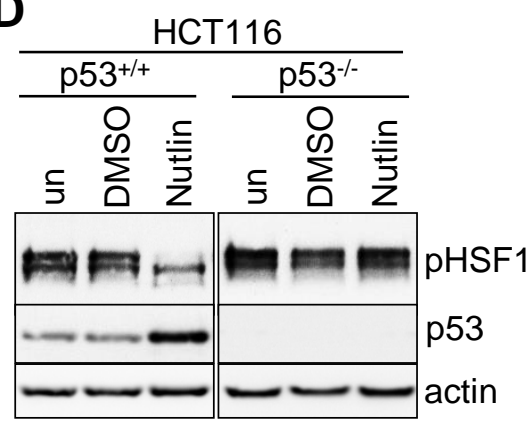

B

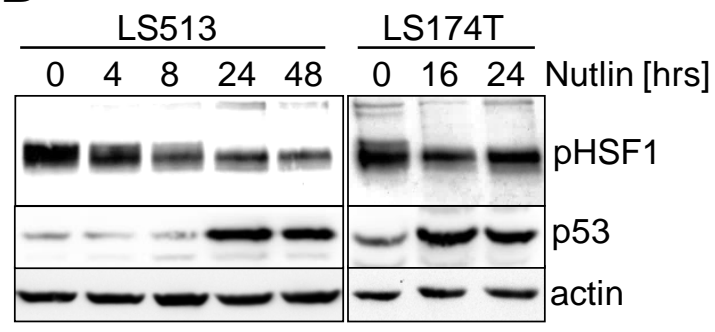

E \begin{tabular}{llllll}
\multicolumn{5}{c}{ SW480 } \\
\cline { 1 - 4 } 0 & 2 & 4 & 8 & 24 & Nutlin [hrs]
\end{tabular}

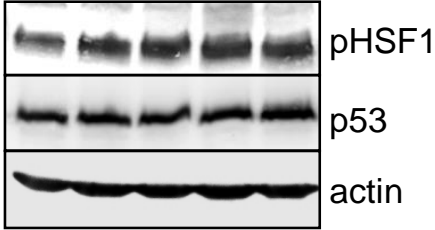

$\mathbf{F}$

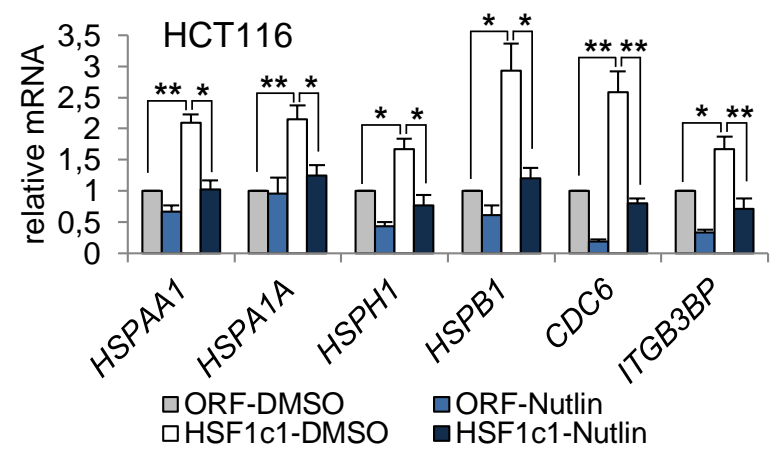

Supp Fig. 3 
A RKO

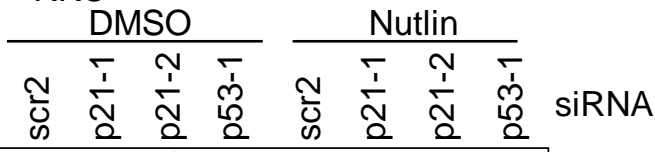

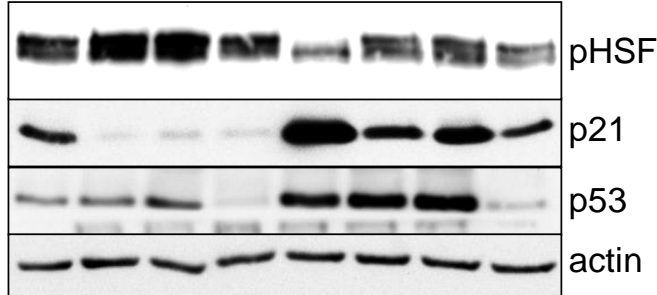

C

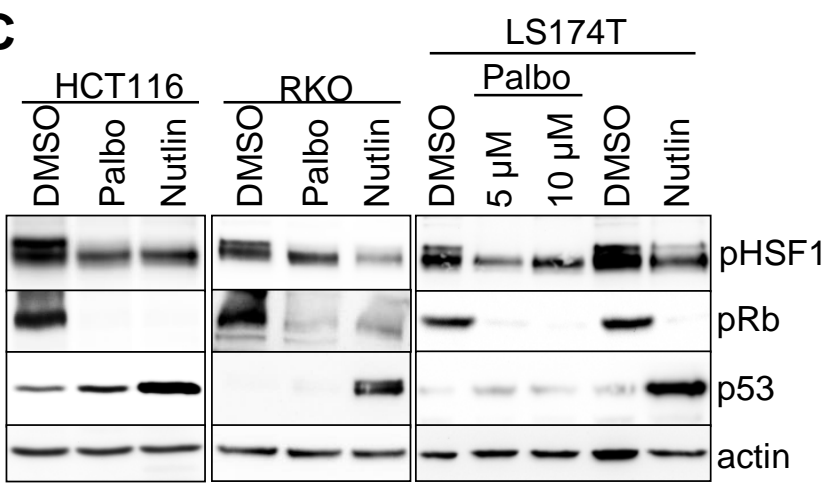

E

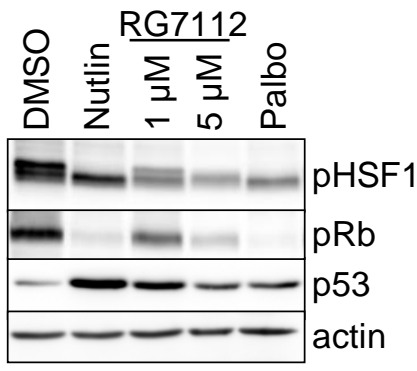

HCT116

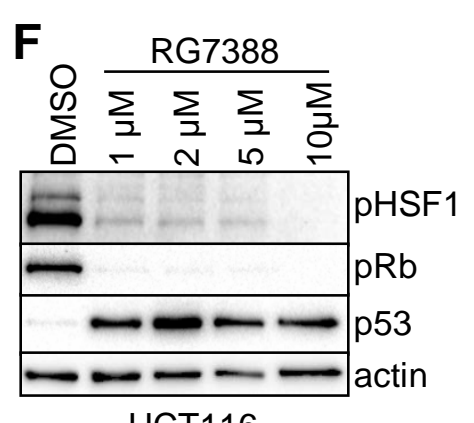

HCT116
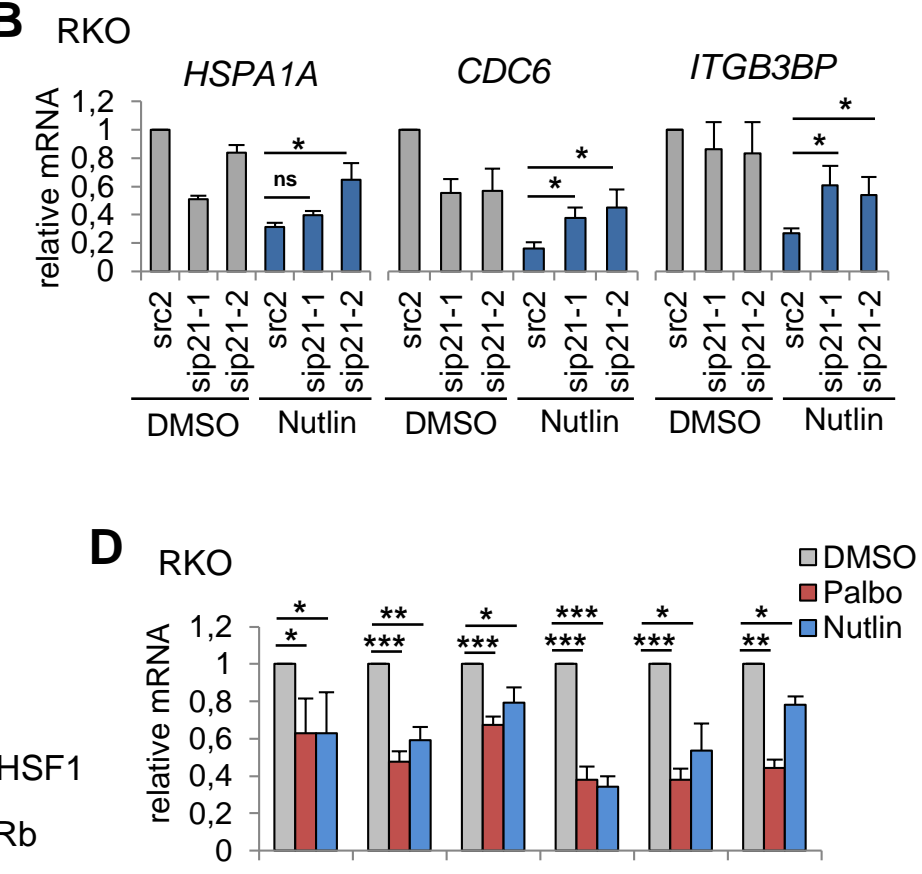

D RKO aDMSO

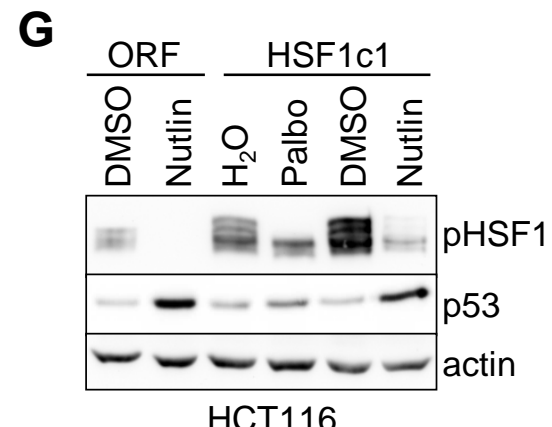

H

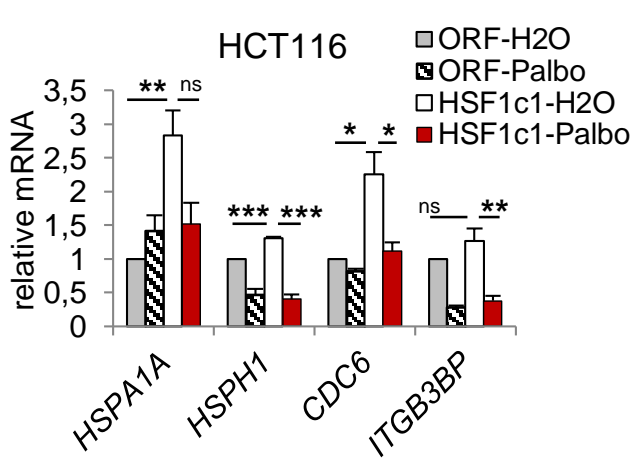

I HCT116 RO3306
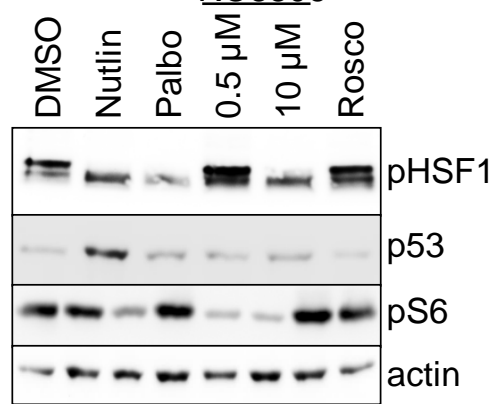

Figure 4 


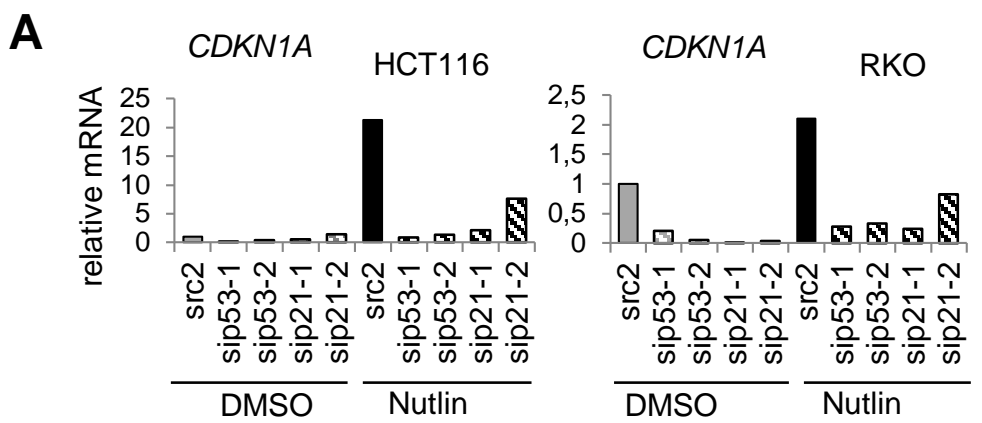

B HCT116

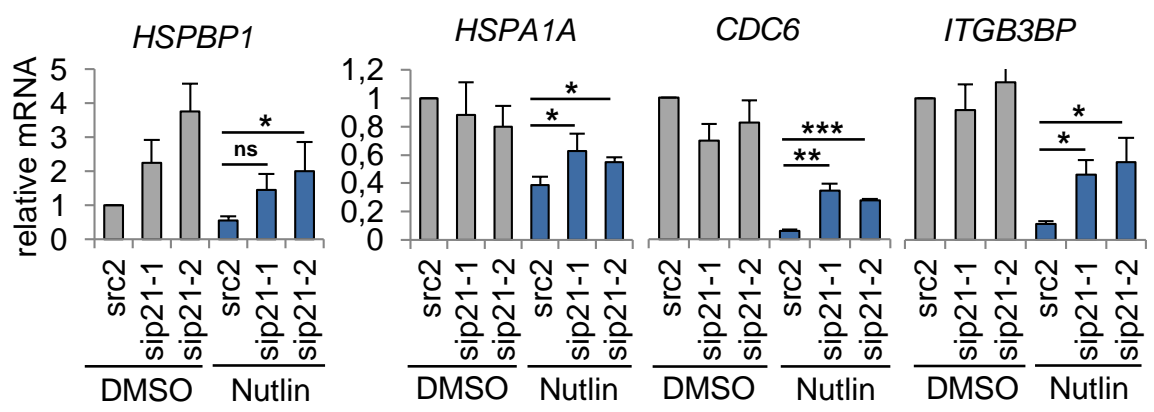

Supp Fig. 4 
A HCT116

B $\frac{\text { HCT116 }}{\text { DMSO Nutlin }}$ RKSO Nutlin
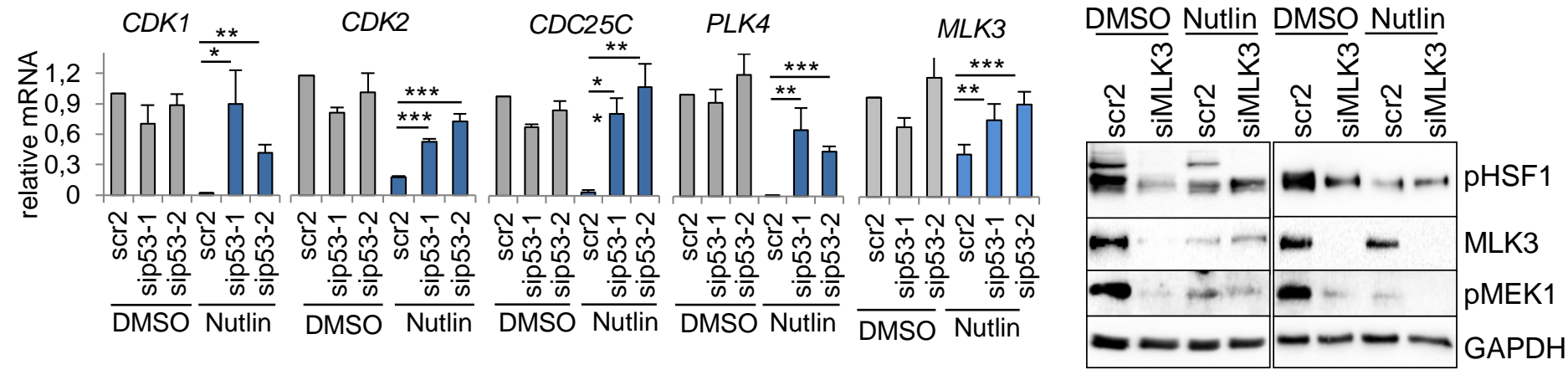

C

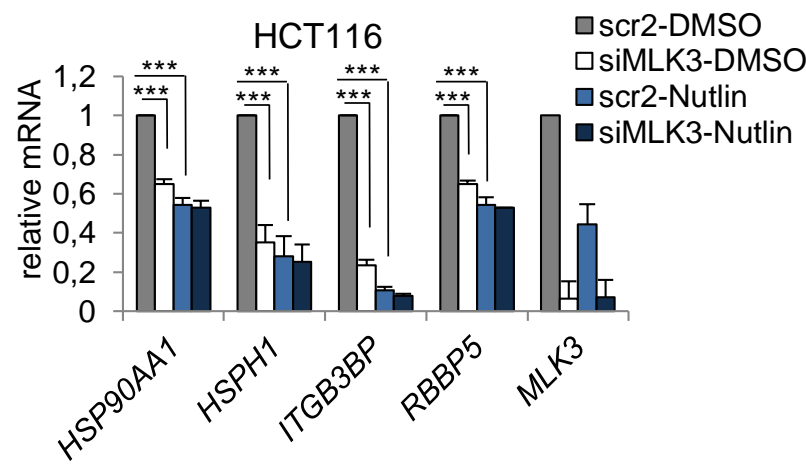

E

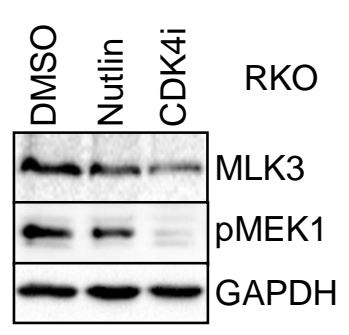

F

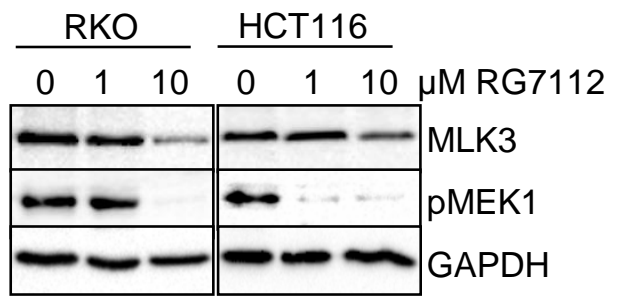

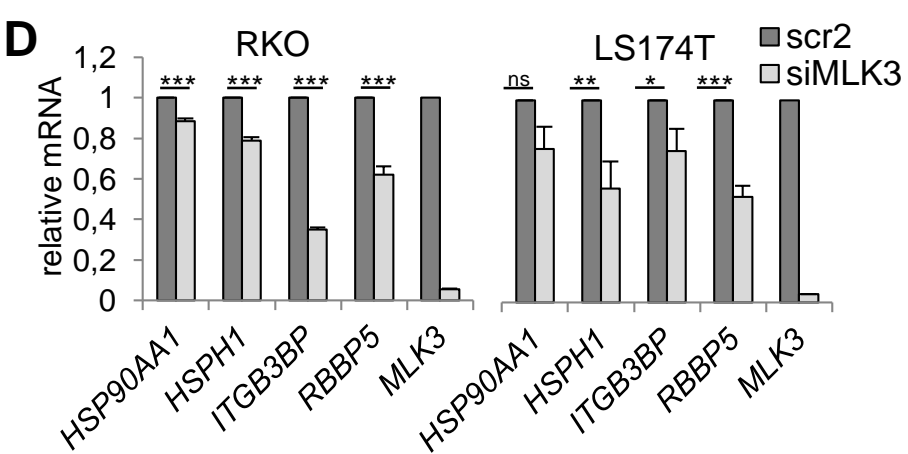

G

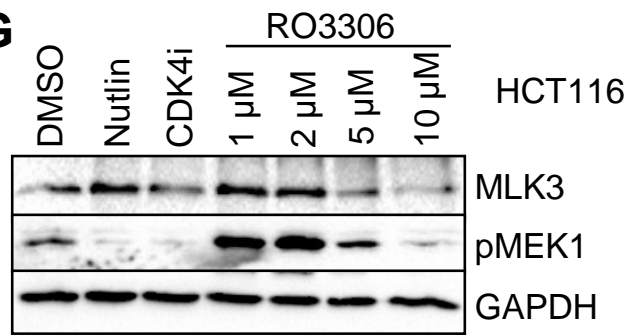

Figure 5 
A
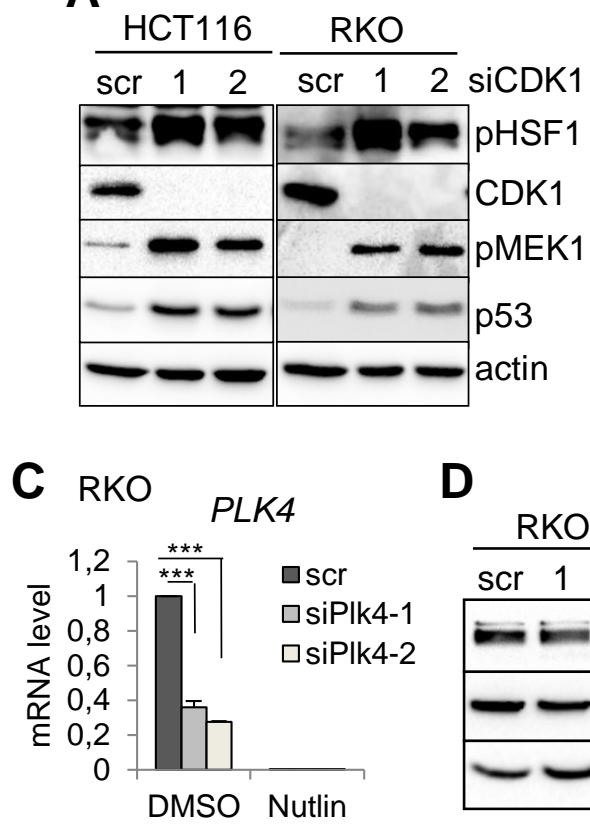

B

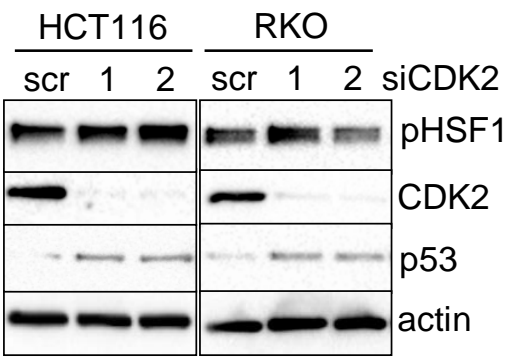

E

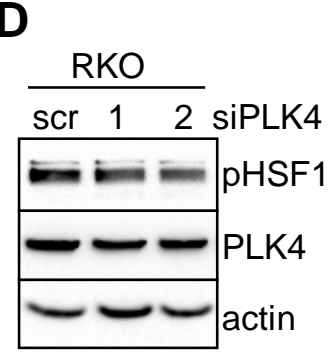

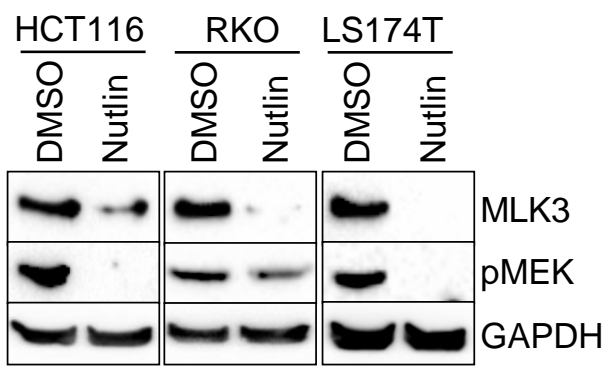

Supp Fig. 5 


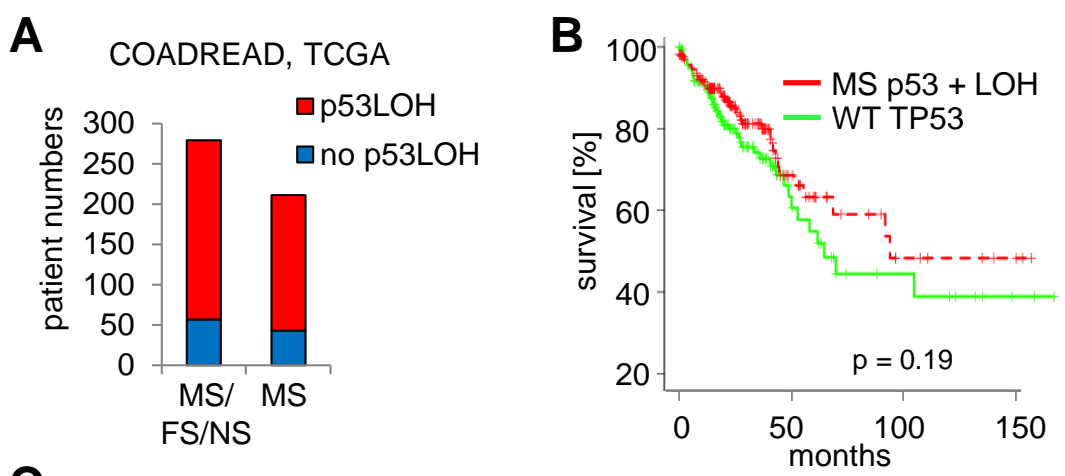

C COADREAD

WT TP53 $(n=213)$

MS + LOH $(n=168)$

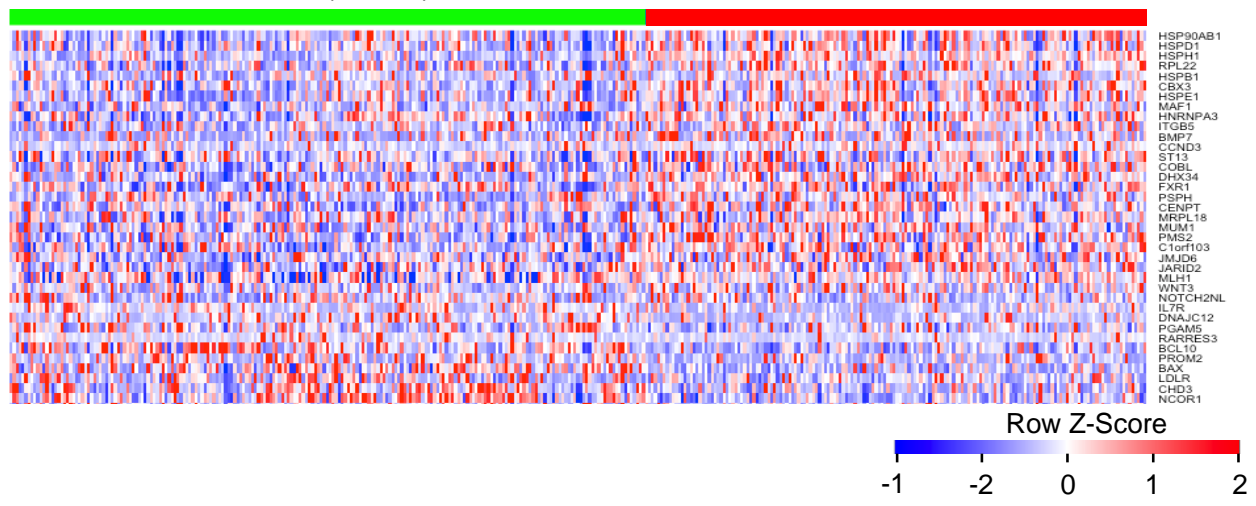

Figure 6 
A COADREAD

WT TP53 $(n=213) \quad$ MS/FS/NS + LOH $(n=222)$
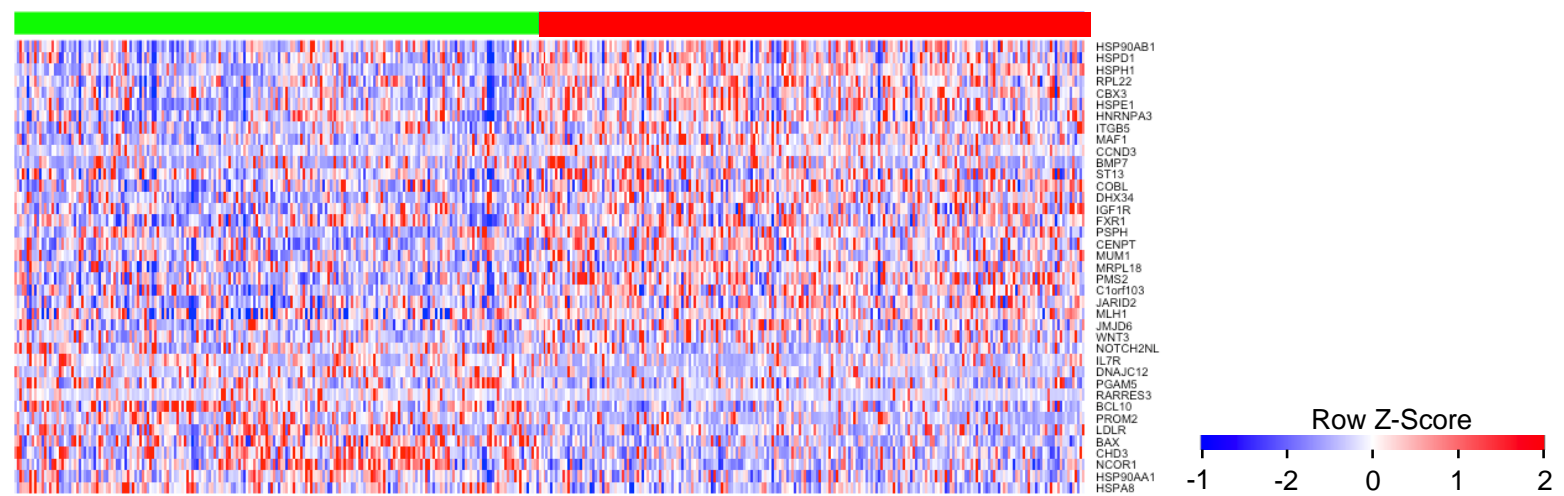

B
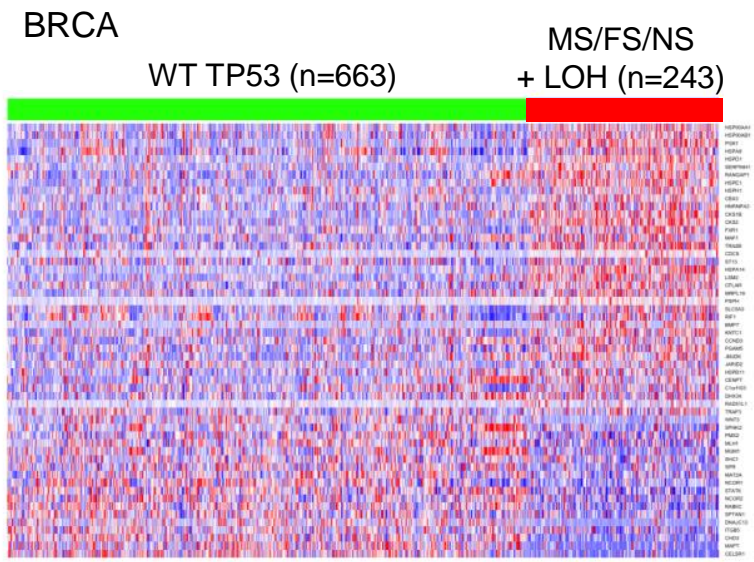

C

$$
\begin{array}{ccc}
\text { BRCA } & & \text { MS }+ \text { LOH } \\
& \text { WT TP53 }(n=663) & (n=166)
\end{array}
$$

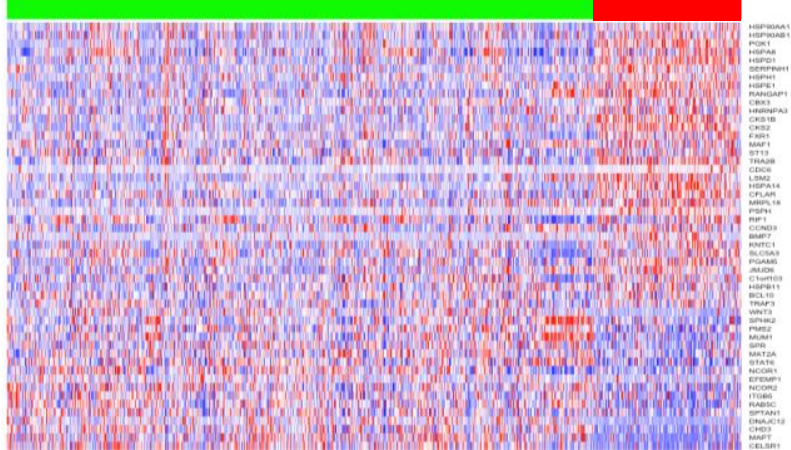

D $\mathrm{MCF7}$

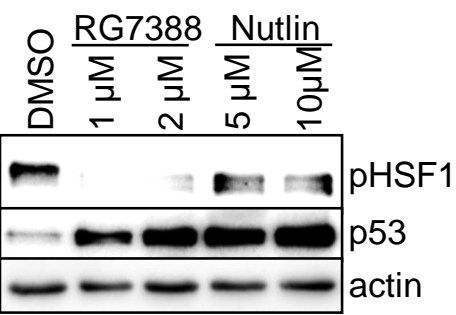

MDA-MB-231

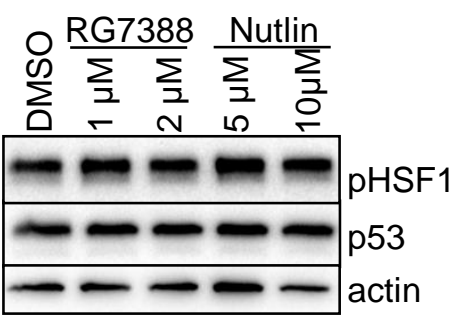


A

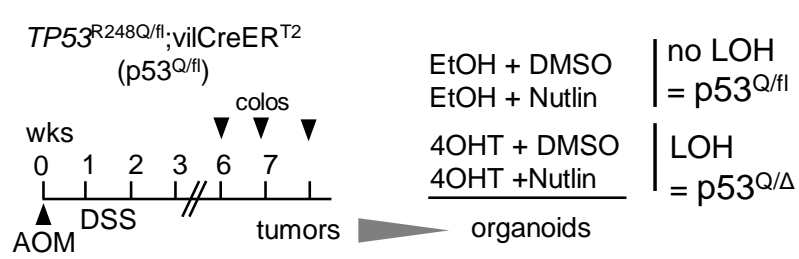

B

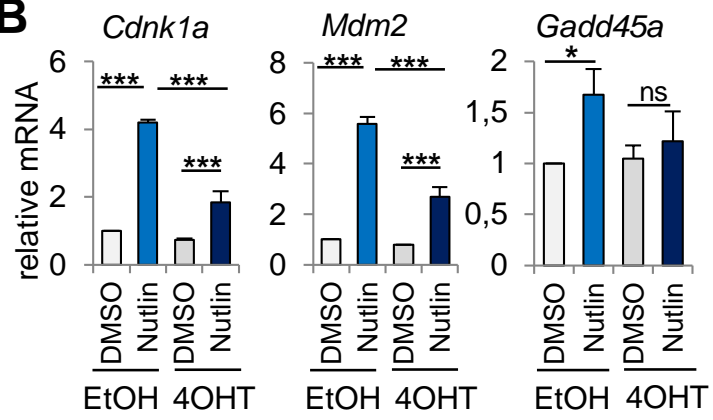

C

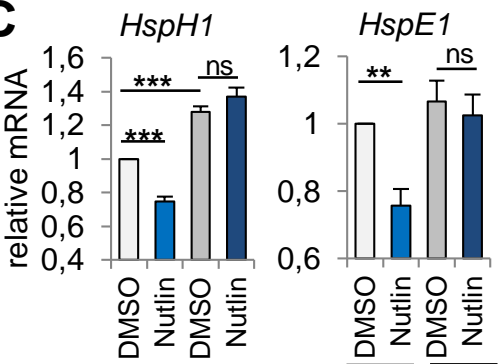

$\mathrm{EtOH} 4 \mathrm{OHT}$

$\mathrm{EtOH} 4 \mathrm{OHT}$

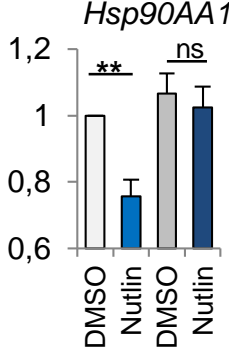

$\mathrm{EtOH} 4 \mathrm{OHT}$ $\operatorname{ltg} b 3 b p$
$* \quad \mathrm{~ns}$

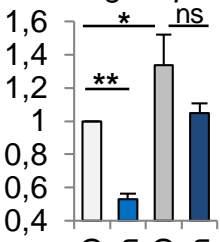

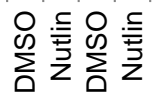

$\mathrm{EtOH} 4 \mathrm{OHT}$

E

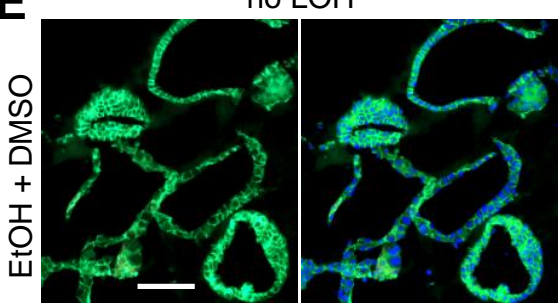

\begin{tabular}{l} 
\\
\hline \\
\hline \multirow{3}{*}{+} \\
+ \\
\hline \\
\hline \\
\hline
\end{tabular}

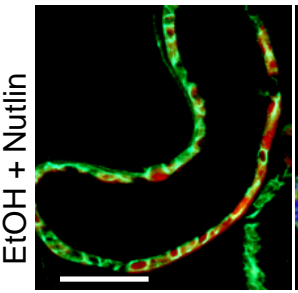

p53 Ecad

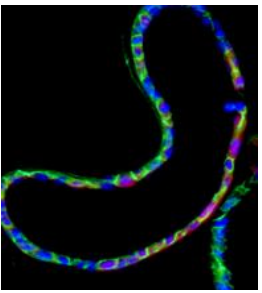

p53 Ecad DAPI
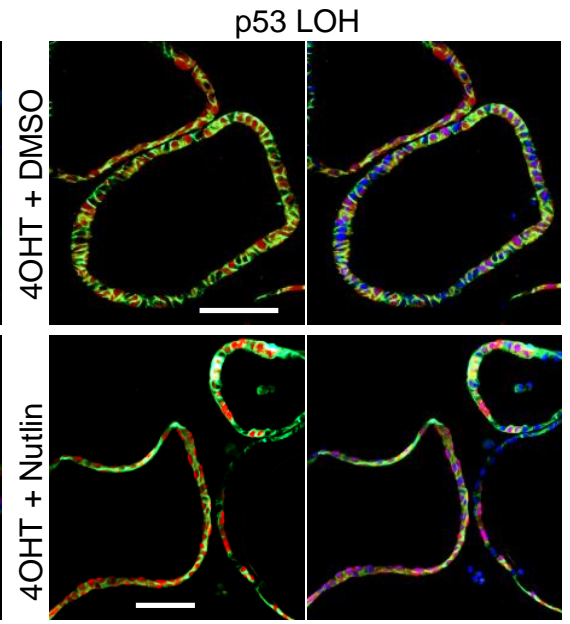

p53 Ecad

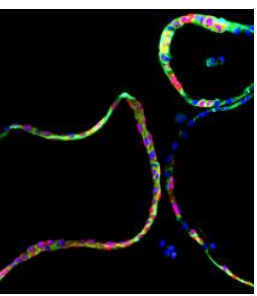

p53 Ecad DAPI
D

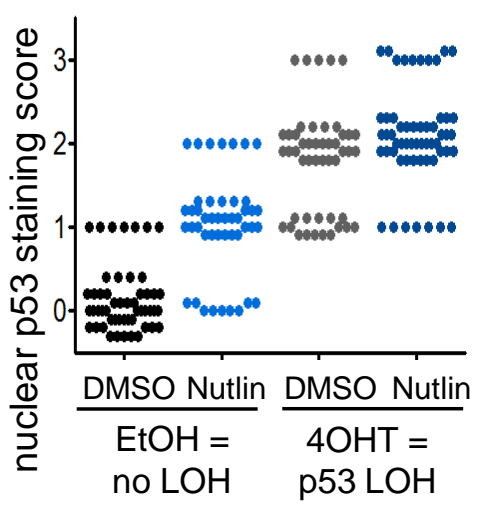

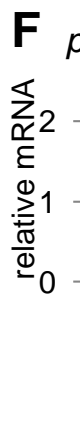

pre-miR34a Vim

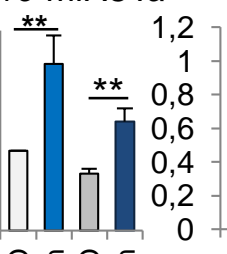

은 은

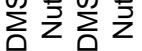

$\mathrm{EtOH} 4 \mathrm{OHT}$

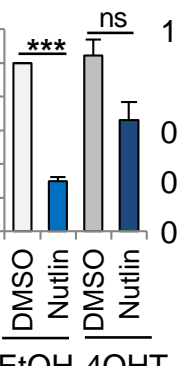

$\mathrm{EtOH} 4 \mathrm{OHT}$
Snai1

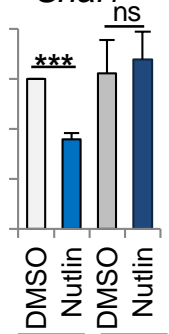

Figure 7 
A

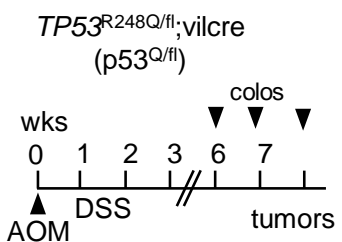

$$
\begin{aligned}
& \begin{array}{l|l}
\mathrm{EtOH}+\mathrm{DMSO} & \text { no LOH } \\
\mathrm{EtOH}+\text { Nutlin } & =\mathrm{p5} 3^{\mathrm{Q} / \mathrm{fl}}
\end{array} \\
& \text { 4-OHT + DMSO } \mathrm{LOH} \\
& \underline{4-\mathrm{OHT}+\text { Nutlin }}=\mathrm{p} 53^{\mathrm{Q} / \mathrm{\Delta}} \\
& \text { organoids }
\end{aligned}
$$

B
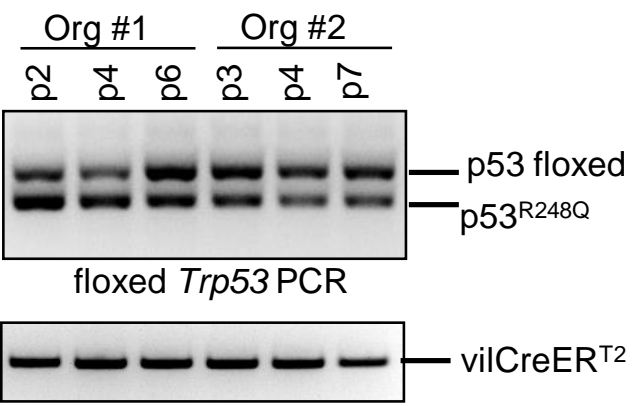

villincreER ${ }^{\mathrm{T} 2} \mathrm{PCR}$

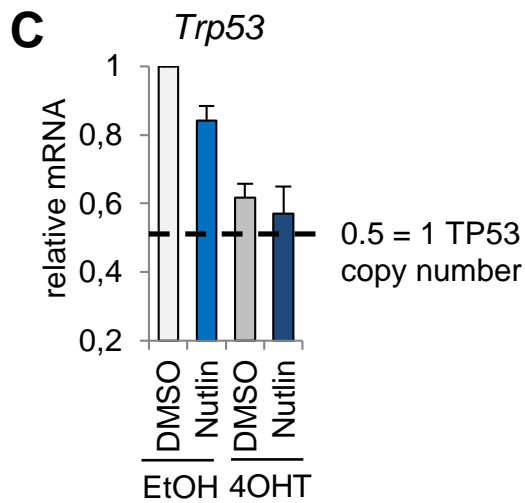

Supp Fig. 7 